\title{
Nonparametric Significance Testing in Measurement Error Models
}

\author{
Hao Dong* and Luke Taylor ${ }^{\dagger}$
}

February 15, 2020

\begin{abstract}
We develop the first nonparametric significance test for regression models with classical measurement error in the regressors. In particular, the Cramér-von Mises test and the Kolmogorov-Smirnov test for the null hypothesis $E\left[Y \mid X^{*}, Z^{*}\right]=E\left[Y \mid X^{*}\right]$ are proposed when only noisy measurements of $X^{*}$ and $Z^{*}$ are available. The asymptotic null distributions of the test statistics are derived and a bootstrap method is implemented to obtain the critical values. Despite the test statistics being constructed using deconvolution estimators, we show that the test can detect a sequence of local alternatives converging to the null at the $\sqrt{n}$-rate. We also highlight the finite sample performance of the test through a Monte Carlo study.
\end{abstract}

\section{Introduction}

R. A. Fisher stated, "in the investigation of living beings by biological methods, statistical tests of significance are essential" (Fisher, 1929, pp. 191). In this paper, we propose the first nonparametric significance test for data contaminated with classical measurement error. The test is flexible in that the measurement error may enter through the variables being tested, the controlling variables, or a combination of both.

Theory often provides little guidance on model specification; in the majority of cases, model choice - linearity in particular - is determined according to simplicity rather than adequacy. Under model misspecification, estimators are generally inconsistent, and consequently, statistical tests which use such estimators have incorrect size. To overcome this problem, many tests, including the one proposed

\footnotetext{
${ }^{*}$ Southern Methodist University, Department of Economics; Email: haod@smu.edu; Address: 3300 Dyer Street, Dallas, TX 75275 , US

${ }^{\dagger}$ Aarhus University, Department of Economics; Email: lntaylor@econ.au.dk; Address: Fuglesangs Allé 4, 8210 Aarhus, Denmark.
} 
in this paper, are conducted using nonparametric methods which impose less stringent conditions on functional form.

Unfortunately, the relaxation of assumptions when using any nonparametric estimator comes at the cost of slower convergence rates. This results in a reduction in power for tests based on such estimators. To remedy this problem, 'non-smoothing' tests were developed which detect local alternatives at the $\sqrt{n}$-rate (see, for example, Bierens, 1982, 1990, and Stute, 1997). This is in contrast to 'smoothing' tests which typically attain slower than $\sqrt{n}$ convergence (see, for example, Härdle and Mammen, 1993). However, smoothing tests have been shown to have advantages over non-smoothing tests in detecting high-frequency alternatives (Fan and Li, 2000; Su and White, 2008), and are asymptotically pivotal. Nonetheless, the level accuracy of smoothing tests which use an approximation of the asymptotic distribution can be quite poor (Härdle and Mammen, 1993) and their performance can depend heavily on the bandwidth when using bootstrap critical values (Lavergne and Vuong, 2000) ${ }^{1}$. As such, we follow a non-smoothing approach in this paper.

There is a plethora of research on nonparametric significance testing when data is uncontaminated (see, for example, Robinson, 1989; Chen and Fan, 1999; Delgado and González-Manteiga, 2001). While, as of yet, there appear to be no results on such tests in the presence of measurement error. However, there has been work carried out on other testing problems in this setting. In particular, model specification testing has received recent attention with Hall and Ma (2007b) proposing a non-smoothing test and Otsu and Taylor (2019) providing results for a smoothing test. Although specification tests share some similar characteristics with significance tests, these tests are fundamentally different in the nature of the null hypothesis. The null model in the case of a specification test is typically parametric (for example, a linear specification), whereas a significance test has a nonparametric null. Indeed, in the majority of non-smoothing specification tests, there is no need for any type of smoothing. In contrast, non-smoothing significance tests still require nonparametric estimation (despite the somewhat misleading name).

There is also a growing literature on the related problem of variable selection in nonparametric and semiparametric models with measurement error (see, for example, Ma and Li, 2010; Zhang, Ma and Carroll, 2017). Although the problem of variable selection is similar to significance testing, the two areas are quite distinct. Variable selection is concerned with finding the most parsimonious model

\footnotetext{
${ }^{1}$ González-Manteiga and Crujeiras (2013) provide a useful review of this vast literature.
} 
while retaining as much predictive ability as possible; this typically involves the use of information criteria such as AIC or BIC. On the other hand, significance tests take no stance on the performance of a given model, they aim only to test the plausibility of a null hypothesis.

The test proposed in this paper uses kernel deconvolution to deal with the measurement error problem. Deconvolution has received considerable attention in recent decades and it is impractical to mention more than just a fraction of the varied work in this literature. Within statistics and econometrics, deconvolution has been used primarily for density estimation (Carroll and Hall, 1988; Stefanski and Carroll, 1990) and estimation of a regression function (Fan and Truong, 1993; Butucea and Taupin, 2008); with this, inference procedures have also been developed (Bissantz, Dümbgen, Holzmann and Munk, 2007; Kato and Sasaki, 2018, 2019). In addition, it is increasingly popular to construct deconvolution methods which do not require a priori knowledge of the measurement error distribution (Delaigle, Hall and Meister, 2008; Hu and Ridder, 2012; Delaigle and Hall, 2016). See Schennach (2016) for a recent review of this growing literature.

This paper is organised as follows. Section 2 outlines the hypothesis of interest and the test statistics, as well as discussing possible alternatives to our test. Section 3 presents the asymptotic properties of our test statistics under the null and the alternative hypothesis, respectively. Section 4 provides a consistent bootstrap procedure to compute critical values. Section 5 relaxes the known error distribution assumption using repeated measurements. Section 6 considers the small sample performance of our test through a Monte Carlo study. Finally, Section 7 concludes. We relegate mathematical proofs of theorems to Appendix A.

\section{Testing Framework}

Let $Y \in \mathbb{R}$ be a response variable and $\left(X^{*}, Z^{*}\right) \in \mathbb{R}^{2}$ be a pair of regressors. We assume that $\left(X^{*}, Z^{*}\right)$ are unobservable due to measurement error, but that we can observe noisy measurements, $X$ and $Z$, generated by

$$
\begin{aligned}
& X=X^{*}+\epsilon, \quad E[\epsilon]=0, \\
& Z=Z^{*}+\nu, \quad E[\nu]=0,
\end{aligned}
$$


where $(\epsilon, \nu)$ is a pair of classical measurement errors, i.e. $\epsilon$ and $\nu$ are independent of $\left(X^{*}, Y, Z^{*}\right)$. Although independence between $(\epsilon, \nu)$ and $\left(X^{*}, Y, Z^{*}\right)$ is assumed, this is not in fact necessary; we only require $f_{X}^{\mathrm{ft}}(t)=f^{\mathrm{ft}}(t) f_{\epsilon}^{\mathrm{ft}}(t)$ and $f_{Z}^{\mathrm{ft}}(t)=f_{Z^{*}}^{\mathrm{ft}}(t) f_{\nu}^{\mathrm{ft}}(t)$ for all $t \in \mathbb{R}$, where $f_{A}$ denotes the density of a random variable $A, f$ denotes the density of $X^{*}$, and $m^{\mathrm{ft}}(t) \equiv \frac{1}{2 \pi} \int e^{\mathrm{i} t u} m(u) d u$ denotes the Fourier transform of a function $m$ with $\mathrm{i}=\sqrt{-1}$.

In this paper, we are interested in testing the significance of $Z^{*}$ when both $X^{*}$ and $Z^{*}$ are mismeasured, i.e. we wish to test the hypothesis

$$
\mathrm{H}_{0}: E\left[Y \mid X^{*}, Z^{*}\right]=E\left[Y \mid X^{*}\right] \quad \text { a.s. }
$$

based on an i.i.d. sample of $(X, Y, Z)$. Notice that the null hypothesis is equivalent to the conditional moment restriction

$$
E\left[\left(Y-E\left[Y \mid X^{*}\right]\right) \mid X^{*}, Z^{*}\right]=0 \quad \text { a.s.. }
$$

In the spirit of Bierens $(1982,1990),(2.3)$ can be written as an unconditional moment restriction of the form

$$
T(\xi) \equiv E\left[\left(Y-r\left(X^{*}\right)\right) f\left(X^{*}\right) \mathcal{W}\left(X^{*}, Z^{*} ; \xi\right)\right]=0 \quad \text { for all } \xi \in \Xi
$$

where $r(x)=E\left[Y \mid X^{*}=x\right], f(x)>0$ a.s., ${ }^{2}$ and $\mathcal{W}(x, z ; \xi)$ is a 'generically totally revealing' function (Stinchcombe and White, 1998) indexed by $\xi \in \Xi$ for some compact set $\Xi \subseteq \mathbb{R}^{2}$ with non-empty interior. Common choices for $\mathcal{W}(x, z ; \xi)$ include $e^{\mathrm{i}(x, z) \xi}$ and $e^{(x, z) \xi}$ used in Bierens (1982, 1990), respectively, and $\mathbb{I}\{(x, z) \leq \xi\}$ proposed by Stute (1997), where $\mathbb{I}\{\cdot\}$ denotes the indicator function. In this paper, we take $\mathcal{W}(x, z ; \xi)=e^{\mathrm{i}(x, z) \xi}$ as this simplifies many of the technical proofs. However, we conjecture that very similar results can be obtained using any generically totally revealing function.

In the error-free case, both $X^{*}$ and $Z^{*}$ are observable, and $T(\xi)$ can be estimated by

$$
\check{T}_{n}(\xi)=\frac{1}{n} \sum_{j=1}^{n}\left(Y_{j}-\check{r}\left(X_{j}^{*}\right)\right) \check{f}\left(X_{j}^{*}\right) e^{\mathrm{i}\left(X_{j}^{*}, Z_{j}^{*}\right) \xi},
$$

where $\check{r}(x)$ is a nonparametric kernel regression estimator of $r(x)$ and $\check{f}(x)$ is a kernel density estimator

\footnotetext{
${ }^{2}$ The multiplication by $f\left(X^{*}\right)$ in the definition of $T(\xi)$ is used only to remove the random denominator in $E\left[Y \mid X^{*}\right]$ and hence simplify the analysis.
} 
of $f(x)$, as in Delgado and González-Manteiga (2001). In our case, $\check{T}_{n}(\xi)$ is infeasible as $X_{j}^{*}$ and $Z_{j}^{*}$ are unobservable due to measurement error. However, $T(\xi)$ can still be estimated by evaluating $r(x)$, $f(x)$, and the joint density $h(x, y, z)$ of $\left(X^{*}, Y, Z^{*}\right)$ in the expression

$$
T(\xi)=\iiint(y-r(x)) f(x) h(x, y, z) e^{\mathrm{i}(x, z) \xi} d x d y d z
$$

using deconvolution methods.

In particular, $r(x), f(x)$, and $h(x, y, z)$ can be estimated by

$$
\begin{gathered}
\hat{r}(x)=\frac{\sum_{j=1}^{n} Y_{j} \mathbb{K}_{\epsilon}\left(\frac{x-X_{j}}{b_{n}}\right)}{\sum_{j=1}^{n} \mathbb{K}_{\epsilon}\left(\frac{x-X_{j}}{b_{n}}\right)}, \quad \hat{f}(x)=\frac{1}{n b_{n}} \sum_{j=1}^{n} \mathbb{K}_{\epsilon}\left(\frac{x-X_{j}}{b_{n}}\right), \\
\hat{h}(x, y, z)=\frac{1}{n b_{n}^{3}} \sum_{j=1}^{n} K\left(\frac{y-Y_{j}}{b_{n}}\right) \mathbb{K}_{\epsilon}\left(\frac{x-X_{j}}{b_{n}}\right) \mathbb{K}_{\nu}\left(\frac{z-Z_{j}}{b_{n}}\right),
\end{gathered}
$$

where the deconvolution kernel associated with a random error $\eta$, denoted as $\mathbb{K}_{\eta}$, is defined as

$$
\mathbb{K}_{\eta}(u)=\frac{1}{2 \pi} \int e^{-\mathrm{i} t u} \frac{K^{\mathrm{ft}}(t)}{f_{\eta}^{\mathrm{ft}}\left(t / b_{n}\right)} d t
$$

with a standard kernel function $K$ and a bandwidth $b_{n}$.

Substituting these estimators into (2.4) leads to the following estimator for $T(\xi)$

$$
\begin{aligned}
T_{n}(\xi) & =\iiint(y-\hat{r}(x)) \hat{f}(x) \hat{h}(x, y, z) e^{\mathrm{i}(x, z) \xi} d x d y d z \\
& =\frac{1}{n^{2} b_{n}^{4}} \sum_{j=1}^{n} \sum_{k=1}^{n} \iint\left\{\begin{array}{c}
\mathbb{K}_{\epsilon}\left(\frac{x-X_{j}}{b_{n}}\right) \mathbb{K}_{\epsilon}\left(\frac{x-X_{k}}{b_{n}}\right) \mathbb{K}_{\nu}\left(\frac{z-Z_{k}}{b_{n}}\right) e^{\mathrm{i}(x, z) \xi} \\
\times \int\left(y-Y_{j}\right) K\left(\frac{y-Y_{k}}{b_{n}}\right) d y
\end{array}\right\} d x d z \\
& =\frac{1}{n^{2} b_{n}^{3}} \sum_{j=1}^{n} \sum_{k=1}^{n} \iint\left\{\begin{array}{c}
\mathbb{K}_{\epsilon}\left(\frac{x-X_{j}}{b_{n}}\right) \mathbb{K}_{\epsilon}\left(\frac{x-X_{k}}{b_{n}}\right) \mathbb{K}_{\nu}\left(\frac{z-Z_{k}}{b_{n}}\right) e^{\mathrm{i}(x, z) \xi} \\
\times \int\left(Y_{k}-Y_{j}+b_{n} y\right) K(y) d y
\end{array}\right\} d x d z \\
& =\frac{1}{n^{2} b_{n}^{3}} \sum_{j \neq k}^{n}\left(Y_{k}-Y_{j}\right) \iint \mathbb{K}_{\epsilon}\left(\frac{x-X_{j}}{b_{n}}\right) \mathbb{K}_{\epsilon}\left(\frac{x-X_{k}}{b_{n}}\right) \mathbb{K}_{\nu}\left(\frac{z-Z_{k}}{b_{n}}\right) e^{\mathrm{i}(x, z) \xi} d x d z,
\end{aligned}
$$

where the third equality follows by a change of variables and the last equality follows from $\int K(u) d u=$ 1 and $\int u K(u) d u=0$.

Note that smoothing along $Y$ is not needed in the construction of $T_{n}(\xi)$, even though it is used 
in the construction of $\hat{h}$. Also note that the same bandwidth is used within each kernel function; however, this is only to keep the notation simple and is not necessary. In Section 6, we discuss a bandwidth selection scheme that can be applied individually to each bandwidth and which we use in the analysis of the finite sample performance of our test. Finally, $f_{\epsilon}$ and $f_{\nu}$ are assumed to be known in the construction of $T_{n}(\xi)$, which rarely holds in practice. In Section 5, we show how an additional noisy measurement of $\left(X^{*}, Z^{*}\right)$ can be used to relax this known error distribution assumption, and prove the properties of the resulting test.

The test statistic is constructed as a continuous functional of $T_{n}(\xi)$. In this paper, we consider two popular examples of such functionals: the Cramér-von Mises-type and Kolmogorov-Smirnov-type functionals. Corresponding test statistics are given by

$$
\begin{aligned}
C M_{n} & =\int_{\Xi}\left|T_{n}(\xi)\right|^{2} d \xi, \\
K S_{n} & =\sup _{\xi \in \Xi}\left|T_{n}(\xi)\right| .
\end{aligned}
$$

Throughout this paper, to keep the notation simple, we focus on the case of only two regressors which are both affected by measurement error. The proposed test, however, can easily be adapted to the general multivariate case with a combination of error-free and contaminated regressors. For example, when $X^{*}=\left(X_{1}^{*}, \ldots, X_{d_{x}}^{*}\right)$ is correctly measured and $Z^{*}=\left(Z_{1}^{*}, \ldots, Z_{d_{z}}^{*}\right)$ is unobservable due to measurement, let $Z=\left(Z_{1}, \ldots, Z_{d_{z}}\right)$ be a noisy measurement of $Z^{*}$ generated by $Z_{d}=Z_{d}^{*}+\nu_{d}$ for $d=1, \ldots, d_{z}$. Following a similar route to the bivariate fully contaminated case, the test statistic can be constructed as $C M_{n}^{\prime}=\int_{\Xi}\left|T_{n}^{\prime}(\xi)\right|^{2} d \xi$ or $K S_{n}^{\prime}=\sup _{\xi \in \Xi}\left|T_{n}^{\prime}(\xi)\right|$, where

$$
T_{n}^{\prime}(\xi)=\frac{1}{n^{2} b_{n}^{3}} \sum_{j \neq k}\left(Y_{k}-Y_{j}\right) \iint\left\{\begin{array}{c}
\prod_{d=1}^{d_{x}} K\left(\frac{x_{d}-X_{d, j}^{*}}{b_{n}}\right) \prod_{d=1}^{d_{x}} K\left(\frac{x_{d}-X_{d, k}^{*}}{b_{n}}\right) \\
\times \prod_{d=1}^{d_{z}} \mathbb{K}_{\nu_{d}}\left(\frac{z_{d}-Z_{d, k}}{b_{n}}\right) e^{\mathrm{i}\left(x^{\prime}, z^{\prime}\right) \xi}
\end{array}\right\} d x d z
$$

with $x=\left(x_{1}, \ldots, x_{d_{x}}\right)^{\prime}, z=\left(z_{1}, \ldots, z_{d_{z}}\right)^{\prime}$, and $\xi=\left(\xi_{1}, \ldots, \xi_{d_{x}+d_{z}}\right)^{\prime}$. We conjecture that analogous results to our main theorems can be established for such cases.

It is important to emphasise that conducting a conventional nonparametric significance test using the mismeasured regressors would, in general, lead to misleading results. To see this, note that by 
ignoring the measurement error, a conventional test would work with a null hypothesis of the form

$$
\mathrm{H}_{0}^{\prime}: E[Y \mid X, Z]=E[Y \mid X] \quad \text { a.s.. }
$$

However, $\mathrm{H}_{0}^{\prime}$ fails under $\mathrm{H}_{0}$ unless $\int E\left[Y \mid X^{*}=x\right]\left[f_{X^{*} \mid X, Z}(x)-f_{X^{*} \mid X}(x)\right] d x=0$, which would fail if $f_{X^{*} \mid X, Z} \neq f_{X^{*} \mid X}$. Hence, a conventional test which ignores measurement error has, in general, incorrect size.

There are, however, special cases where the measurement error can indeed be ignored and a conventional test using the contaminated sample can be conducted. One such example is when $Z^{*}$ is independent of $X^{*}$. In such a situation, $f_{X^{*} \mid X, Z}=f_{X^{*} \mid X}$ given $(\epsilon, \nu)$ are classical, and $\mathrm{H}_{0}^{\prime}$ holds if and only if $\mathrm{H}_{0}$ is true. A conventional test can then be conducted without accounting for the measurement error issue.

A second example occurs when we wish to test the joint significance of all regressors. In such a situation, we test $E\left[Y \mid Z^{*}\right]=E[Y]$, which is equivalent to testing $E[Y \mid Z]=E[Y]$. Hence, the test constructed in this paper is applicable when there is interest in testing the significance of a nontrivial subset of regressors, which are not independent of other regressors, and when the regressors are contaminated with classical measurement error.

A potential alternative to our test could be constructed by linearising the model by taking, for example, a finite polynomial in the regressors, estimating the regression coefficients using an IV approach, and conducting a Wald test. Note that an instrument is required in this case: typically a repeated measurement of the mismeasured regressor. Furthermore, for each transformation of each variable, the instrument is the analogous transformation of the repeated measurement. Of course, this is a simplification of the model and, although being valid, would not lead to a consistent test if the model is misspecified. However, for practical purposes, we may hope that this approach fares well. Unfortunately, as our simulation results in Section 6 indicate, this is not the case, in general. The reason is twofold. Nonlinear transformations of mismeasured regressors generally exacerbate the measurement error problem. Additionally, the strength of an instrument typically deteriorates with nonlinear transformations.

Finally, it is also worth noting that while our test is able to accommodate discrete regressors if they are correctly measured, discrete mismeasured variables cannot be accounted for as they would violate the classical measurement error assumption. 


\section{Asymptotic Theory}

In this section, the asymptotic distributions of the test statistics $C M_{n}$ and $K S_{n}$ are established under the null hypothesis $\mathrm{H}_{0}$ and under a sequence of local alternatives $\mathrm{H}_{1 n}$ that converge to $\mathrm{H}_{0}$ at the parametric rate $n^{-1 / 2}$, respectively. Let $g(x)=r(x) f(x)$ and $g_{X^{*}, Z^{*}}(x, z)=E\left[Y \mid X^{*}=x, Z^{*}=\right.$

$z] f_{X^{*}, Z^{*}}(x, z)$. Also, for a function $m: \mathbb{R}^{d} \rightarrow \mathbb{R}$, denote $m^{\left(\delta_{1}, \ldots, \delta_{d}\right)}(u)=\frac{\partial^{\delta_{1}+\cdots+\delta_{d m}(u)}}{\partial u_{1}^{\delta_{1} \ldots \partial u_{d}^{\delta_{d}}}}$, where $d$ is any positive integer, $u=\left(u_{1}, \ldots, u_{d}\right)$ is a $d$-dimensional vector, and $\left(\delta_{1}, \ldots, \delta_{d}\right)$ is a $d$-dimensional vector of nonnegative integers.

\subsection{Asymptotic null distribution}

To derive the asymptotic null distributions of the test statistics $C M_{n}$ and $K S_{n}$, we first impose the following regularity conditions.

\section{Assumption D.}

(i) $\left\{X_{j}, Y_{j}, Z_{j}\right\}_{j=1}^{n}$ is an i.i.d. sample of $(X, Y, Z)$ satisfying (2.1) and (2.2) with $E|Y|^{2}<\infty$.

(ii) $(\epsilon, \nu)$ are mutually independent and are independent of $\left(X^{*}, Y, Z^{*}\right)$ with characteristic functions $f_{\epsilon}^{\mathrm{ft}}(t) \neq 0$ and $f_{\nu}^{\mathrm{ft}}(t) \neq 0$ for all $t \in \mathbb{R}$. Also, $\inf _{\xi \in \Xi}\left|f_{\nu}^{\mathrm{ft}}\left(\xi_{2}\right)\right|>\underline{c}$ for a positive constant $\underline{c}$.

(iii) $b_{n} \rightarrow 0$ as $n \rightarrow \infty$.

Assumption D (i) requires random sampling and the existence of the second moment of $Y$. Assumption D (ii) is standard in the classical measurement error literature. The assumption $f_{\epsilon}^{\mathrm{ft}}(t) \neq 0$ and $f_{\nu}^{\mathrm{ft}}(t) \neq 0$ for all $t \in \mathbb{R}$ is needed for kernel based deconvolution methods and is satisfied by many conventional distributions; however, a notable exception is the uniform distribution, whose characteristic function is oscillatory and has infinitely many zeros. If this assumption is unlikely to hold, a possible solution involves using a ridge parameter approach (see, for example, Hall and Meister, 2007, and Meister, 2009). Given $f_{\nu}^{\mathrm{ft}}$ is nonvanishing anywhere and $\Xi$ is compact, the assumption $\inf _{\xi \in \Xi}\left|f_{\nu}^{\mathrm{ft}}\left(\xi_{2}\right)\right|>\underline{c}$ is innocuous, and it is imposed here for regularity purposes. Assumption D (iii) simply states that the bandwidth must decay to zero as the sample size grows.

As is typical in the nonparametric measurement error literature, we consider two separate cases characterised by bounds on the decay rate of the tail of the characteristic function of the measurement errors. For the ordinary smooth case, where the error characteristic function decays at a polynomial rate, the following assumptions are imposed. 


\section{Assumption O.}

(i) $f, g, f_{X^{*}, Z^{*}}$, and $g_{X^{*}, Z^{*}}$ are $p$-times continuously differentiable with bounded and integrable derivatives, where $p$ is a positive integer satisfying $p>1+2 \alpha$, and satisfy $E\left[Y^{2}\left|f^{(l)}(X)\right|^{2}\right]<\infty$, $E\left|g^{(l)}(X)\right|^{2}<\infty, E\left[Y^{2}\left|f_{X^{*}, Z^{*}}^{(l, 0)}(X)\right|^{2}\right]<\infty$, and $E\left|g_{X^{*}, Z^{*}}^{(l, 0)}(X)\right|^{2}<\infty$ for $0 \leq l \leq p$, and

$$
\begin{aligned}
& \left|f^{(p)}(x+c)-f^{(p)}(x)\right|<m_{1, p}(x)|c|, \\
& \left|g^{(p)}(x+c)-g^{(p)}(x)\right|<m_{2, p}(x)|c|, \\
& \max _{0 \leq l \leq p}\left|f_{X^{*}, Z^{*}}^{(l, p-l)}\left(x+c_{x}, z+c_{z}\right)-f_{X^{*}, Z^{*}}^{(l, p-l)}(x, z)\right|<m_{3, p}(x, z) \sqrt{c_{x}^{2}+c_{z}^{2}}, \\
& \max _{0 \leq l \leq p}\left|g_{X^{*}, Z^{*}}^{(l, p-l)}\left(x+c_{x}, z+c_{z}\right)-g_{X^{*}, Z^{*}}^{(l, p-l)}(x, z)\right|<m_{4, p}(x, z) \sqrt{c_{x}^{2}+c_{z}^{2}},
\end{aligned}
$$

for some bounded and integrable functions $m_{1, p}(x), m_{2, p}(x), m_{3, p}(x, z)$, and $m_{4, p}(x, z)$.

(ii) The characteristic function of $\epsilon$ is of the form

$$
f_{\epsilon}^{\mathrm{ft}}(t)=\frac{1}{c_{0}^{\mathrm{os}}+c_{1}^{\mathrm{os}} t+\cdots+c_{\alpha}^{\mathrm{os}} t^{\alpha}}
$$

for all $t \in \mathbb{R}$ and for some finite constants $c_{0}^{\mathrm{os}}, \ldots, c_{\alpha}^{\mathrm{os}}$ with $c_{0}^{\mathrm{os}} \neq 0$, and $\alpha>0$.

(iii) $K$ is differentiable to order $p$ and satisfies

$$
\int K(u) d u=1, \quad \int u^{p+1} K(u) d u \neq 0, \quad \int u^{l} K(u) d u=0, \text { for } l=1,2, \ldots, p .
$$

Also, $K^{\mathrm{ft}}$ is compactly supported on $[-1,1]$, symmetric around zero, and bounded.

(iv) $n b_{n}^{2 p} \rightarrow 0$ and $n b_{n}^{2+4 \alpha} \rightarrow \infty$ as $n \rightarrow \infty$.

(v) For $c_{l}^{\mathrm{os}}\left(\xi_{1}\right)=(-\mathrm{i})^{l} \sum_{h=l}^{\alpha} c_{h}^{\mathrm{os}}\left(\begin{array}{l}l \\ h\end{array}\right) \xi_{1}^{h-l}$, we have

$$
E\left[\begin{array}{c}
\sup _{\xi \in \Xi}\left|\sum_{l=0}^{\alpha} c_{l}^{\mathrm{os}}\left(\xi_{1}\right) \int\left\{g_{X^{*}, Z^{*}}^{(l, 0)}-Y f_{X^{*}, Z^{*}}^{(l, 0)}\right\}(X, z) e^{\mathrm{i}(X, z) \xi} d z\right|^{2} \\
+\sup _{\xi \in \Xi}\left|\sum_{l=0}^{\alpha} c_{l}^{\mathrm{os}}\left(\xi_{1}\right)\left\{g^{(l)}-Y f^{(l)}\right\}(X)\right|^{2}
\end{array}\right]<\infty
$$

Assumption O (i) imposes further conditions on the existence of moments, and gives smoothness restrictions on the structural functions, which are equivalent to Assumption 3 and Assumption 5 in Powell, Stock and Stoker (1989). 
Assumption $\mathrm{O}$ (ii) is an ordinary smooth condition. Specifically, it requires that $f_{\epsilon}^{\mathrm{ft}}(t)$ decays to zero at a polynomial rate as $|t| \rightarrow \infty$. Examples of densities that are ordinary smooth include the Laplace and gamma distributions. Compared to the conventional ordinary smooth assumption, which only imposes bounds on the tail behaviour of the error characteristic function, Assumption $\mathrm{O}$ (ii) is stronger in the sense that it characterises the exact limiting behaviour of $f_{\epsilon}^{\mathrm{ft}}(t)$ as $|t| \rightarrow \infty$, which is necessary to characterise the asymptotic distribution of our test statistic. It is also worth noting that Assumption $\mathrm{O}$ (ii) only concerns $f_{\epsilon}$, while $f_{\nu}$ is left unrestricted except for the nonvanishing nature of $f_{\nu}^{\mathrm{ft}}$ anywhere and $\inf _{\xi \in \Xi}\left|f_{\nu}^{\mathrm{ft}}\left(\xi_{2}\right)\right|>\underline{c}$ as required by Assumption D. This is because our test statistic is constructed asymmetrically with respect to $X$ and $Z$. In particular, as $Z$ only appears under the alternative, due to the presence of the generically totally revealing function $e^{\mathrm{i}(x, z) \xi}$ and integration with respect to $z$, to characterise the asymptotic distributions of the test statistics, we only need to characterise the behaviour of $f_{\nu}^{\mathrm{ft}}$ over a compact set. In contrast, $X$ appears in both the null and alternative model. Thus, even after considering the presence of the generically totally revealing function $e^{\mathrm{i}(x, z) \xi}$ and integration with respect to $x$, the exact limiting behaviour of $f_{\epsilon}^{\mathrm{ft}}(t)$ as $|t| \rightarrow \infty$ is still needed to characterise the asymptotic distribution of the test statistic. An implication of this insight is that if only regressors which are being tested are contaminated with error, our test would be adaptive in the sense that the asymptotic distribution of our test statistic can be characterised in a uniform way irrespective of the smoothness of the error distribution.

Assumption $\mathrm{O}$ (iii) requires a higher-order kernel to remove the asymptotic bias of the nonparametric estimators and is used in the proof of Lemma 3. Besides removing the estimation bias, the higher-order kernel is also used to characterise the integration involving the deconvolution kernel in Lemma 5. Higher-order kernels are common in deconvolution problems; indeed, Meister (2009) discusses how kernels of any order can be constructed quite simply.

The first condition of Assumption $\mathrm{O}$ (iv) is required to ensure that the bias from the nonparametric estimators is asymptotically negligible and the second condition is needed for the error of the Hájek projection to be asymptotically negligible. Note that Assumption $\mathrm{O}$ (iv) implicitly requires $p>$ $1+2 \alpha$, i.e. the structural functions have to be smooth enough to use the Hájek projection while undersmoothing, which is guaranteed by Assumption $\mathrm{O}$ (i).

Finally, Assumption $\mathrm{O}(\mathrm{v})$ is a high-level assumption on the boundedness of the asymptotic variance of $T_{n}(\xi)$; an analogous assumption is made in Fan (1995). 
To derive the asymptotic distributions of $C M_{n}$ and $K S_{n}$ under the null hypothesis $\mathrm{H}_{0}$ for the ordinary smooth case, we need to characterise the limiting behaviour of the empirical process $T_{n}(\xi)$.

Theorem 1. Suppose Assumptions $D$ and $O$ hold. Then, under $\mathrm{H}_{0}$, we have

$$
\sqrt{n} T_{n}(\xi) \Rightarrow G^{\mathrm{os}}(\xi)
$$

where $G^{\mathrm{os}}(\xi)$ is a Gaussian process with mean zero and covariance structure $\operatorname{Var}\left[r_{\infty}^{\mathrm{os}}(X, Y, Z ; \xi)\right]$ for $r_{\infty}^{\mathrm{os}}(X, Y, Z ; \xi)=\sum_{l=0}^{\alpha} c_{l}^{\mathrm{os}}\left(\xi_{1}\right)\left[\left\{Y f^{(l)}-g^{(l)}\right\}(X) e^{\mathrm{i}(X, Z) \xi} f_{\nu}^{\mathrm{ft}-1}\left(\xi_{2}\right)+\int\left\{g_{X^{*}, Z^{*}}^{(l, 0)}-Y f_{X^{*}, Z^{*}}^{(l, 0)}\right\}(X, z) e^{\mathrm{i}(X, z) \xi} d z\right]$.

Based on Theorem 1, using the continuous mapping theorem (see, for example, van der Vaart and Wellner, 1996, Theorem 1.3.6), the asymptotic null distributions of $C M_{n}$ and $K S_{n}$ for the ordinary smooth case are established as follow.

Corollary 1. Suppose Assumptions $D$ and $O$ hold. Then, under $\mathrm{H}_{0}$, we have

$$
\begin{aligned}
& n C M_{n} \stackrel{d}{\rightarrow} \int_{\Xi}\left|G^{\mathrm{os}}(\xi)\right|^{2} d \xi, \\
& \sqrt{n} K S_{n} \stackrel{d}{\rightarrow} \sup _{\xi \in \Xi}\left|G^{\mathrm{os}}(\xi)\right| .
\end{aligned}
$$

Corollary 1 shows that in the ordinary smooth case, the Cramér-von Mises statistic and KolmogorovSmirnov statistic converge to the squared $L_{2}$-norm and the Skorohod norm of a Gaussian process, respectively, which are characterised by the limit of the empirical process $T_{n}(\xi)$. For the Cramér-von Mises statistic $C M_{n}$, following Bierens and Ploberger (1997), the limiting distribution can be further characterised as an infinite weighted sum of independent chi-squared random variables.

It is worth noting that unlike typical results based on deconvolution estimation, $T_{n}(\xi)$ achieves a $\sqrt{n}$-rate. This is, however, not the first time that a parametric rate has been obtained for functionals of nonparametric estimators in a measurement error context. Zhu and Cui (2003) and Hall and Ma (2007a) develop semiparametric estimators for regression functions which also achieve a $\sqrt{n}$-rate of convergence.

For the second case, known as supersmooth measurement error, where the error characteristic function decays in an exponential rate, the following assumptions are imposed. 


\section{Assumption S.}

(i) $f, g, f_{X^{*}, Z^{*}}$, and $g_{X^{*}, Z^{*}}$ are infinitely differentiable.

(ii) The characteristic function of $\epsilon$ is of the form

$$
f_{\epsilon}^{\mathrm{ft}}(t)=c^{\mathrm{ss}} e^{-\mu|t|^{\gamma}},
$$

for all $t \in \mathbb{R}$ and for some positive constant $c^{\mathrm{ss}}, \mu$, and positive even integer $\gamma$.

(iii) $K$ is infinitely differentiable and satisfies

$$
\int K(u) d u=1, \quad \int u^{l} K(u) d u=0, \text { for all } l \in \mathbb{N} .
$$

Also, $K^{\mathrm{ft}}$ is compactly supported on $[-1,1]$, symmetric around zero, and bounded.

(iv) $n b_{n}^{2} e^{-\mu 2^{\gamma+2} b_{n}^{-\gamma}} \rightarrow \infty$ as $n \rightarrow \infty$.

(v) For $c_{l}^{\mathrm{ss}}\left(\xi_{1}\right)=(-\mathrm{i})^{l} \sum_{h=\lceil l / \gamma\rceil}^{\infty} \frac{\mu^{h}}{c^{\mathrm{ss} h !}}\left(\begin{array}{c}l \\ h \gamma\end{array}\right) \xi_{1}^{h \gamma-l}$, we have

$$
E\left[\begin{array}{c}
\sup _{\xi \in \Xi}\left|\sum_{l=0}^{\infty} c_{l}^{\mathrm{ss}}\left(\xi_{1}\right) \int\left\{g_{X^{*}, Z^{*}}^{(l, 0)}-Y f_{X^{*}, Z^{*}}^{(l, 0)}\right\}(X, z) e^{\mathrm{i}(X, z) \xi} d z\right|^{2} \\
+\sup _{\xi \in \Xi}\left|\sum_{l=0}^{\infty} c_{l}^{\mathrm{ss}}\left(\xi_{1}\right)\left\{g^{(l)}-Y f^{(l)}\right\}(X)\right|^{2}
\end{array}\right]<\infty
$$

Assumption S (i) is restrictive but appears necessary when dealing with supersmooth measurement error. However, the conditions on the density functions simply require the distributions of the latent regressors to be supersmooth themselves. Indeed, Meister (2009) discusses how the class of infinitely differentiable functions still contain a 'comprehensive nonparametric class of densities' (pp. 44). For the conditional mean functions, there are still a broad class of functions which satisfy such a smoothness restriction, including - but not limited to - all polynomials, circular functions, exponentials, and products or sums of such functions.

Assumption S (ii) is a supersmooth condition requiring $f_{\epsilon}^{\mathrm{ft}}(t)$ to decay to zero at an exponential rate as $|t| \rightarrow \infty$. The most common example of a density satisfying this assumption is the Gaussian density where $\gamma=2$. The constraint on $\gamma$ being even also seems necessary in this setup; specifically, it is required in the proof of Lemma 4 (the supersmooth case). Note that this restriction rules out the Cauchy distribution; Van Es and Uh (2005) and Kato and Sasaki (2018) find similar issues with the 
Cauchy distribution in a supersmooth error setting and also make this assumption. Similarly to the ordinary smooth case, Assumption S (ii) is stronger than the conventional supersmooth assumption in the sense that it not only imposes bounds on the tail behaviour of the error characteristic function, but also characterises the exact limiting behaviour of $f_{\epsilon}^{\mathrm{ft}}(t)$ as $|t| \rightarrow \infty$. Also, similarly to the ordinary smooth case, due to the asymmetry of our test statistics with respect to $X$ and $Z$, the smoothness restriction only concerns $f_{\epsilon}$.

Assumption S (iii) requires $K$ to be an infinite-order kernel so that it can adapt to the smoothness of $f, g, f_{X^{*}, Z^{*}}$, and $g_{X^{*}, Z^{*}}$, as used in the proof of Lemma 6 . A common choice of $K$ satisfying this restriction is the flat-top kernel (McMurry and Politis, 2004).

As a result of the infinite-order kernel removing the bias in the nonparametric estimator, in contrast to Assumption O (iv) for the ordinary smooth case, Assumption S (iv) does not impose an upper bound on the bandwidth, which is usually required to control the magnitude of the estimation bias. Similar to Assumption O (iv) for the ordinary smooth case, a lower bound on the bandwidth is imposed in Assumption S (iv). In particular, Assumption S (iv) is satisfied by a bandwidth of order $\ln (n)^{-1 / \gamma}$.

Finally, Assumption S (v) is a high-level assumption on the boundedness of the asymptotic variance of $T_{n}(\xi)$, which is analogous to Assumption $\mathrm{O}(\mathrm{v})$ for the ordinary smooth case.

The limiting behaviour of the empirical process $T_{n}(\xi)$ under the null hypothesis $\mathrm{H}_{0}$ for the supersmooth case is given in the following theorem.

Theorem 2. Suppose Assumptions $D$ and $S$ hold. Then, under $\mathrm{H}_{0}$, we have

$$
\sqrt{n} T_{n}(\xi) \Rightarrow G^{\mathrm{ss}}(\xi)
$$

where $G^{\mathrm{ss}}(\xi)$ is a Gaussian process with mean zero and covariance structure $\operatorname{Var}\left[r_{\infty}^{\mathrm{ss}}(X, Y, Z ; \xi)\right]$ for $r_{\infty}^{\mathrm{ss}}(X, Y, Z ; \xi)=\sum_{l=0}^{\infty} c_{l}^{\mathrm{ss}}\left(\xi_{1}\right)\left[\left\{Y f^{(l)}-g^{(l)}\right\}(X) e^{\mathrm{i}(X, Z) \xi} f_{\nu}^{\mathrm{ft}-1}\left(\xi_{2}\right)+\int\left\{g_{X^{*}, Z^{*}}^{(l, 0)}-Y f_{X^{*}, Z^{*}}^{(l, 0)}\right\}(X, z) e^{\mathrm{i}(X, z) \xi} d z\right]$

Based on Theorem 2, using the continuous mapping theorem, the asymptotic null distributions of $C M_{n}$ and $K S_{n}$ for the supersmooth case are established as follow. 
Corollary 2. Suppose Assumptions $D$ and $S$ hold. Then, under $\mathrm{H}_{0}$, we have

$$
\begin{aligned}
& n C M_{n} \stackrel{d}{\rightarrow} \int_{\Xi}\left|G^{\mathrm{ss}}(\xi)\right|^{2} d \xi, \\
& \sqrt{n} K S_{n} \stackrel{d}{\rightarrow} \sup _{\xi \in \Xi}\left|G^{\mathrm{ss}}(\xi)\right| .
\end{aligned}
$$

Corollary 2 includes parallel results for the supersmooth case to the results in Corollary 1. Fan (1995) derived an average derivative estimator using deconvolution kernel techniques and went on to prove the $\sqrt{n}$-convergence of the estimator. Our derivation of the limits of the empirical process $T_{n}(\xi)$ in both Theorem 1 and 2 follows a similar route as in Fan (1995) due to the link between average derivative estimators and non-smoothing tests. However, Fan (1995) was restricted to the ordinary smooth case. The key to our result for the supersmooth case lies in Lemma 4, where we show that supersmooth error can be thought of as an ordinary smooth problem with an infinite smoothness parameter.

\section{$3.2 \quad$ Asymptotic power}

In this section, we analyse the power properties of the test against a sequence of local alternatives $\mathrm{H}_{1 n}$ that converges to $\mathrm{H}_{0}$ at the parametric rate $n^{-1 / 2}$. In particular, we assume a local linear alternative of the form

$$
\mathrm{H}_{1 n}: E\left[Y \mid X^{*}, Z^{*}\right]=E\left[Y \mid X^{*}\right]+\frac{\Delta\left(X^{*}, Z^{*}\right)}{\sqrt{n}} \text { a.s., }
$$

where $\Delta: \mathbb{R}^{2} \rightarrow \mathbb{R}$ is a bounded non-zero function. The local power properties of $C M_{n}$ and $K S_{n}$ under $\mathrm{H}_{1 n}$ can be derived based on the following results.

Theorem 3. Under $\mathrm{H}_{1 n}$, if Assumptions $D$ and $O$ hold, we have

$$
\sqrt{n} T_{n}(\xi) \Rightarrow G^{\mathrm{os}}(\xi)+\mu_{\Delta}(\xi)
$$

and if Assumptions $D$ and $S$ hold, we have

$$
\sqrt{n} T_{n}(\xi) \Rightarrow G^{\mathrm{ss}}(\xi)+\mu_{\Delta}(\xi)
$$

where $G^{\mathrm{os}}(\xi)$ and $G^{\mathrm{ss}}(\xi)$ are the same Gaussian processes as defined in Theorem 1 and 2 , respectively, 
and $\mu_{\Delta}(\xi)$ is a deterministic shift function given by $\mu_{\Delta}(\xi)=E\left[\Delta\left(X^{*}, Z^{*}\right) f\left(X^{*}\right) e^{\mathrm{i}\left(X^{*}, Z^{*}\right) \xi}\right]$.

Theorem 3 shows that under both ordinary smooth and supersmooth measurement error, the asymptotic behaviour of $T_{n}(\xi)$ under $\mathrm{H}_{1 n}$ can be characterised by the same Gaussian process used to characterise the asymptotic behaviour of $T_{n}(\xi)$ under $\mathrm{H}_{0}$ plus a deterministic shift function $\mu_{\Delta}(\xi)$, which essentially characterises the extra bias brought by $\mathrm{H}_{1 n}$. Using the continuous mapping theorem, tests based on a continuous functional of $T_{n}(\xi)$ will have non-trivial power against local alternatives drifting at the rate $n^{-1 / 2}$.

\section{Computation of Critical Values}

The asymptotic distributions derived in Theorems 1 and 2 can be used to obtain critical values. However, as explained in Bierens and Ploberger (1997), the limiting distributions depend on the covariance function which in turn depends on the underlying distribution of the data. As such, the asymptotic distributions are case dependent and challenging to estimate in practice. Given this difficulty, we implement a bootstrap procedure.

Measurement error models provide a challenge for bootstrap procedures because neither the true regressor nor the measurement error is observable. Any residual-based bootstrap approach is infeasible in a measurement error context since the true regressors are needed to construct the residuals. It is possible to follow an approach similar to Hall and Ma (2007b): estimating the density of the true regressor and measurement error using deconvolution techniques, applying a wild bootstrap approach for the regression error, and sampling from these respective densities. However, the estimated densities suffer from the slow rates of convergence associated with deconvolution estimation, and the approach is computationally expensive. In addition, the choice of several tuning parameters is needed. Instead, we consider a simple empirical bootstrap approach.

We focus on the bootstrap for the $K S_{n}$ test statistic but conjecture that very similar results can be obtained for the $C M_{n}$ statistic. A bootstrap sample $\left\{X_{j}^{\#}, Y_{j}^{\#}, Z_{j}^{\#}\right\}_{j=1}^{n}$ is constructed by independently drawing with replacement from the set of observed $\left\{X_{j}, Y_{j}, Z_{j}\right\}_{j=1}^{n}$ and the bootstrap statistic is calculated as $\sqrt{n} K S_{n}^{\#}=\sup _{\xi \in \Xi}\left|\sqrt{n}\left\{T_{n}^{\#}(\xi)-E\left[T_{n}^{\#}(\xi)\right]\right\}\right|$, where

$$
T_{n}^{\#}(\xi)=\frac{1}{n^{2} b_{n}^{3}} \sum_{j \neq k}^{n}\left(Y_{k}^{\#}-Y_{j}^{\#}\right) \iint \mathbb{K}_{\epsilon}\left(\frac{x-X_{j}^{\#}}{b_{n}}\right) \mathbb{K}_{\epsilon}\left(\frac{x-X_{k}^{\#}}{b_{n}}\right) \mathbb{K}_{\nu}\left(\frac{z-Z_{k}^{\#}}{b_{n}}\right) e^{\mathrm{i}(x, z) \xi} d x d z
$$


To analyse the properties of this procedure, we strengthen Assumptions O (v) and S (v) as follows.

\section{Assumption B.}

$E\left[\int_{\Xi} r_{\infty}^{\mathrm{os}}(X, Y, Z ; \xi)^{4} d \xi\right]<\infty$ holds under Assumption $O$ or $E\left[\int_{\Xi} r_{\infty}^{\mathrm{ss}}(X, Y, Z ; \xi)^{4} d \xi\right]<\infty$ holds under Assumption $S$.

Assumptions B places bounds on the fourth moment of the dominant term from the Hájek projection of our empirical process. This assumption is needed to apply Chernozhukov, Chetverikov and Kato (2016, Theorems 2.1 and 2.3).

Define $\hat{c}_{\alpha}$ as the $(1-\alpha)^{\text {th }}$ quantile of $K S_{n}^{\#}$. The validity of the proposed empirical bootstrap procedure is then given in the following theorem.

Theorem 4. Suppose Assumptions $D, O / S$, and $B$ hold, then under $H_{0}$,

$$
P\left\{K S_{n} \leq \hat{c}_{\alpha}\right\} \geq 1-\alpha-o(1)
$$

Theorem 4 provides the asymptotic validity of the empirical bootstrap for our Kolmogorov-Smirnov statistic and shows that quantiles of the bootstrap statistic distribution conditional on the data can be used as critical values for our test. Similar results for the empirical bootstrap in a measurement error setting were found by Kato and Sasaki $(2018,2019)$ in the construction of confidence bands for density estimation and nonparametric regression, respectively, and by Adusumilli, Kurisu, Otsu and Whang (2019) for confidence bands for the cumulative distribution function.

\section{Case of Unknown Measurement Error Distribution}

In this section, we discuss how to relax the known error distribution assumption. Suppose that, in addition to $X$ and $Z$, we also observe $X^{r}$ and $Z^{r}$, which are generated by

$$
\begin{gathered}
X^{r}=X^{*}+\epsilon^{r}, \\
Z^{r}=Z^{*}+\nu^{r},
\end{gathered}
$$

where $\left(\epsilon^{r}, \nu^{r}\right)$ are independent and identically distributed as $(\epsilon, \nu)$. Furthermore, we assume the following. 


\section{Assumption R.}

(i) $\left\{X_{j}^{r}, Z_{j}^{r}\right\}_{j=1}^{n}$ is an i.i.d. sample of $\left(X^{r}, Z^{r}\right)$ satisfying (5.1) and (5.2).

(ii) $\left(\epsilon^{r}, \nu^{r}\right)$ are independent and identically distributed as $(\epsilon, \nu)$, and $f_{\epsilon}^{\mathrm{ft}}$ and $f_{\nu}^{\mathrm{ft}}$ are real-valued.

Assumption $\mathrm{R}$ (ii) requires that $\left(\epsilon^{r}, \nu^{r}\right)$ is an independent copy of $(\epsilon, \nu)$, and $f_{\epsilon}$ and $f_{\nu}$ are symmetric around zero, which is conventional in the deconvolution literature when the error density is unknown (see, for example, Delaigle, Hall and Meister, 2008).

Under Assumption R, $f_{\epsilon}^{\mathrm{ft}}$ and $f_{\nu}^{\mathrm{ft}}$ can be estimated by (Delaigle, Hall and Meister, 2008)

$$
\hat{f}_{\epsilon}^{\mathrm{ft}}(t)=\left|\frac{1}{n} \sum_{j=1}^{n} \cos \left\{t\left(X_{j}-X_{j}^{r}\right)\right\}\right|^{1 / 2}, \quad \hat{f}_{\nu}^{\mathrm{ft}}(t)=\left|\frac{1}{n} \sum_{j=1}^{n} \cos \left\{t\left(Z_{j}-Z_{j}^{r}\right)\right\}\right|^{1 / 2} .
$$

Let $\hat{\mathbb{K}}_{\epsilon}$ and $\hat{\mathbb{K}}_{\nu}$ separately denote the deconvolution kernels based on the estimated error characteristic functions $\hat{f}_{\epsilon}^{\mathrm{ft}}$ and $\hat{f}_{\nu}^{\mathrm{ft}}$, and are defined as

$$
\hat{\mathbb{K}}_{\epsilon}(u)=\frac{1}{2 \pi} \int e^{-\mathrm{i} t u} \frac{K^{\mathrm{ft}}(t)}{\hat{f}_{\epsilon}^{\mathrm{ft}}\left(t / b_{n}\right)} d t, \quad \hat{\mathbb{K}}_{\nu}(u)=\frac{1}{2 \pi} \int e^{-\mathrm{i} t u} \frac{K^{\mathrm{ft}}(t)}{\hat{f}_{\nu}^{\mathrm{ft}}\left(t / b_{n}\right)} d t .
$$

By replacing $\mathbb{K}_{\epsilon}$ and $\mathbb{K}_{\nu}$ by $\hat{\mathbb{K}}_{\epsilon}$ and $\hat{\mathbb{K}}_{\nu}$ respectively, in the case when the error distribution is unknown, $T(\xi)$ can be estimated by

$$
\hat{T}_{n}(\xi)=\frac{1}{n^{2} b_{n}^{3}} \sum_{j \neq k}^{n}\left(Y_{k}-Y_{j}\right) \iint \hat{\mathbb{K}}_{\epsilon}\left(\frac{x-X_{j}}{b_{n}}\right) \hat{\mathbb{K}}_{\epsilon}\left(\frac{x-X_{k}}{b_{n}}\right) \hat{\mathbb{K}}_{\nu}\left(\frac{z-Z_{k}}{b_{n}}\right) e^{\mathrm{i}(x, z) \xi} d x d z
$$

and the test statistics are constructed as

$$
\begin{aligned}
\widehat{C M}_{n} & =\int_{\Xi}\left|\hat{T}_{n}(\xi)\right|^{2} d \xi, \\
\widehat{K S}_{n} & =\sup _{\xi \in \Xi}\left|\hat{T}_{n}(\xi)\right| .
\end{aligned}
$$

It should be noted that alternative estimation schemes for $f_{\epsilon}^{\mathrm{ft}}$ and $f_{\nu}^{\mathrm{ft}}$ exist which rely on weaker assumptions than have been made here. In particular, Li and Vuong (1998) use a lemma by Kotlarski (1967) to construct an estimator of an error characteristic function which relaxes the symmetry assumption by still requires independent repeated measurements. While Delaigle and Hall (2016) develop a clever method which does not require repeated measurements, their work requires symmetry 
in the measurement error and asymmetry in the true regressor. A testing procedure based on such estimators can be constructed in an analogous fashion as outlined above; however, the analysis of the properties of such tests is beyond the scope of this paper.

To derive the asymptotic distributions of $\widehat{C M}_{n}$ and $\widehat{K S}_{n}$, as seen in Theorem 1-3, it is suffice to characterise the asymptotic behaviour of $\hat{T}_{n}(\xi)$. Let

$$
\frac{1}{4 \pi} \int\left\{\begin{array}{c}
\left\{1-\frac{\cos \left(t\left(X-X^{r}\right)\right)}{\left|f_{\epsilon}^{\mathrm{ft}}(t)\right|^{2}}\right\}\left\{\begin{array}{c}
f^{\mathrm{ft}}(t) g_{X^{*}, Z^{*}}^{\mathrm{ft}}\left(\xi_{1}-t, \xi_{2}\right)-g^{\mathrm{ft}}(t) f_{X^{*}, Z^{*}}^{\mathrm{ft}}\left(\xi_{1}-t, \xi_{2}\right) \\
+f^{\mathrm{ft}}\left(\xi_{1}-t\right) g_{X^{*}, Z^{*}}^{\mathrm{ft}}\left(t, \xi_{2}\right)-g^{\mathrm{ft}}\left(\xi_{1}-t\right) f_{X^{*}, Z^{*}}^{\mathrm{ft}}\left(t, \xi_{2}\right)
\end{array}\right\} d t . \\
+\left\{1-\frac{\cos \left(t\left(Z-Z^{r}\right)\right)}{\left|f_{\nu}^{\mathrm{ft}}\left(\xi_{2}\right)\right|^{2}}\right\}\left\{\begin{array}{l}
\mathrm{ft} \\
f^{\mathrm{ft}}(t) g_{X^{*}, Z^{*}}^{\mathrm{ft}}\left(\xi_{1}-t, \xi_{2}\right)-g^{\mathrm{ft}}(t) f_{X^{*}, Z^{*}}^{\mathrm{ft}}\left(\xi_{1}-t, \xi_{2}\right)
\end{array}\right\}
\end{array}\right\}
$$

In the ordinary smooth case, to characterise the asymptotic behaviour of $\hat{T}_{n}(\xi)$, we further impose the following assumptions in addition to Assumption O.

\section{Assumption O'.}

(i) $n b_{n}^{2+12 \alpha} \log \left(b_{n}^{-1}\right)^{-2} \rightarrow \infty$ as $n \rightarrow \infty$.

(ii) $E\left[\int_{\Xi} \hat{r}_{\infty}^{\mathrm{os}}\left(X, X^{r}, Y, Z, Z^{r} ; \xi\right)^{2} d \xi\right]<\infty$, where

$$
\hat{r}_{\infty}^{\mathrm{os}}\left(X, X^{r}, Y, Z, Z^{r} ; \xi\right)=r_{\infty}^{\mathrm{os}}(X, Y, Z ; \xi)+\hat{r}_{\infty}^{\epsilon, \nu}\left(X, X^{r}, Y, Z, Z^{r} ; \xi\right)
$$

Assumption O' (i) is a stronger bandwidth condition than Assumption O (iv), and is required to ensure that the higher order terms in the error brought by the estimation of the error characteristic functions is asymptotically negligible. Assumption O' (ii) is a high-level assumption on the boundedness of the asymptotic variance of $\hat{T}_{n}(\xi)$, as Assumption $\mathrm{O}(\mathrm{v})$ for $T_{n}(\xi)$. In particular, the first term $r_{\infty}^{\mathrm{os}}(X, Y, Z ; \xi)$ characterises the estimation error when the error distribution is known, and the additional term $\hat{r}_{\infty}^{\epsilon, \nu}\left(X, X^{r}, Y, Z, Z^{r} ; \xi\right)$ characterises the main terms in the error brought by the estimation of the error characteristic functions. The following theorem summaries asymptotic behaviour of $\hat{T}_{n}(\xi)$ for the ordinary smooth case under the null hypothesis $\mathrm{H}_{0}$ and under the alternative hypothesis $\mathrm{H}_{1 n}$.

Theorem 5. Suppose Assumptions D, O, R, and O' hold.

(i) Under $\mathrm{H}_{0}$, we have

$$
\sqrt{n} \hat{T}_{n}(\xi) \Rightarrow \hat{G}^{\mathrm{os}}(\xi)
$$


where $\hat{G}^{\mathrm{os}}(\xi)$ denotes a Gaussian process with mean zero and covariance structure $\operatorname{Var}\left[\hat{r}_{\infty}^{\mathrm{os}}\left(X, X^{r}, Y, Z, Z^{r} ; \xi\right)\right]$.

(ii) Under $\mathrm{H}_{1, n}$, we have

$$
\sqrt{n} \hat{T}_{n}(\xi) \Rightarrow \hat{G}^{\mathrm{os}}(\xi)+\mu_{\Delta}(\xi),
$$

where $\mu_{\Delta}(\xi)$ is the same deterministic shift function as defined in Theorem 3.

Theorem 5 shows that in ordinary smooth case when the error distribution is unknown, $\hat{T}_{n}(\xi)$ converges at a $\sqrt{n}$-rate to a Gaussian process $\hat{G}^{\text {os }}(\xi)$, which, by the continuous mapping theorem, implies that the Cramér-von Mises statistic and Kolmogrov-Smirnov statistic converge to the squared $L_{2}$-norm and sup-norm of the Gaussian process $\hat{G}^{\text {os }}(\xi)$, respectively. The Gaussian process $\hat{G}^{\text {os }}(\xi)$ here, however, is different from $G^{\text {os }}(\xi)$, the limit of the empirical process $T_{n}(\xi)$ in the case when the error distribution is known, as the estimation error of the error characteristic functions is non-negligible. Whereas the bias term does not depend on the error distributions, so the deterministic shift function $\mu_{\Delta}(\xi)$ is the same as that obtained in the case when the error distribution is known.

For the supersmooth case, the following assumptions are needed in addition to Assumption S to characterise the asymptotic behaviour of $\hat{T}_{n}(\xi)$.

\section{Assumption S'.}

(i) $n b_{n}^{2} e^{-12 \mu_{\epsilon} 2^{\gamma} b_{n}^{-\gamma}} \log \left(b_{n}^{-1}\right)^{-2} \rightarrow \infty$ as $n \rightarrow \infty$.

(ii) $E\left[\int_{\Xi} \hat{r}_{\infty}^{\mathrm{os}}\left(X, X^{r}, Y, Z, Z^{r} ; \xi\right)^{2} d \xi\right]<\infty$, where

$$
\hat{r}_{\infty}^{\mathrm{ss}}\left(X, X^{r}, Y, Z, Z^{r} ; \xi\right)=r_{\infty}^{\mathrm{ss}}(X, Y, Z ; \xi)+\hat{r}_{\infty}^{\epsilon, \nu}\left(X, X^{r}, Y, Z, Z^{r} ; \xi\right)
$$

Similar to the ordinary smooth case, Assumption S' (i) requires a stronger bandwidth condition than Assumption S (iv) to ensure that the higher order terms in the error brought by the estimation of the error characteristic functions is asymptotically negligible, and Assumption S' (ii) requires the boundedness of the asymptotic variance of $\hat{T}_{n}(\xi)$. The asymptotic behaviour of $\hat{T}_{n}(\xi)$ for the supersmooth case under the null hypothesis $\mathrm{H}_{0}$ and under the alternative hypothesis $\mathrm{H}_{1 n}$ are summarised in the following theorem.

Theorem 6. Suppose Assumptions D, S, R, and S' hold.

(i) Under $\mathrm{H}_{0}$, we have

$$
\sqrt{n} \hat{T}_{n}(\xi) \Rightarrow \hat{G}^{\mathrm{ss}}(\xi),
$$


where $\hat{G}^{\mathrm{os}}(\xi)$ denotes a Gaussian process with mean zero and covariance structure $\operatorname{Var}\left[\hat{r}_{\infty}^{\mathrm{ss}}\left(X, X^{r}, Y, Z, Z^{r} ; \xi\right)\right]$.

(ii) Under $\mathrm{H}_{1, n}$, we have

$$
\sqrt{n} \hat{T}_{n}(\xi) \Rightarrow \hat{G}^{\mathrm{ss}}(\xi)+\mu_{\Delta}(\xi)
$$

where $\mu_{\Delta}(\xi)$ is the same deterministic shift function as defined in Theorem 3.

Theorem 6 includes parallel results for the supermsooth case to the results in Theorem 5 for the ordinary smooth case. Dong, Otsu, and Taylor (2019) showed that if the error density is symmetric around zero, the asymptotic distribution of the average derivative estimator is the same irrespective of whether the error density is estimated or not. Thus, given the link between average derivative estimators and non-smoothing tests, the conclusions of Theorems 5 and 6 seem somewhat surprising. The reason for the discrepancy between the two results resides in the asymmetric nature of $T(\xi)$ which is not present in the average derivative. In particular, the asymmetry is two-fold. First, $T(\xi)$ is based on $f f_{X^{*}, Z^{*}}$, while the average derivative is based on $f f^{\prime}$. Second, $T(\xi)$ involves a revealing function $e^{\mathrm{i}(x, z) \xi}$ for an arbitrary $\xi \in \Xi$, while the average derivative restricts $\xi=(0,0)$. Due to the asymmetry of $T(\xi)$, even when the error density is symmetric around zero, the estimation error of the error characteristic functions is still non-negligible in our test.

Given the non-negligibility of the estimation error of the error characteristic functions, the empirical bootstrap proposed in Section 4 will be inconsistent; this is in contrast to the results of Kato and Sasaki (2018, 2019) and Adusumilli, Kurisu, Otsu and Whang (2019). Since the measurement errors are unobservable, it is not possible to account for their effect using an empirical bootstrap. However, because the estimator for the error characteristic function is constructed using a sample mean, it is possible to construct a multiplier bootstrap version of each estimator, i.e.

$$
\hat{f}_{\epsilon}^{\mathrm{ft \#}}(t)=\left|\frac{1}{n} \sum_{j=1}^{n} \eta_{1, j} \cos \left\{t\left(X_{j}-X_{j}^{r}\right)\right\}\right|^{1 / 2}, \quad \hat{f}_{\nu}^{\mathrm{ft \#}}(t)=\left|\frac{1}{n} \sum_{j=1}^{n} \eta_{2, j} \cos \left\{t\left(Z_{j}-Z_{j}^{r}\right)\right\}\right|^{1 / 2}
$$

where $\eta_{1}$ and $\eta_{2}$ are i.i.d random variables with unit mean and unit variance. These bootstrap estimates can be plugged into $T_{n}^{\#}(\xi)$ to give a bootstrap statistic for $\hat{T}_{n}(\xi)$, denoted as $\hat{T}_{n}^{\#}(\xi)$. However, it is beyond the scope of this paper to prove the consistency of this bootstrap procedure. 


\section{Simulation}

Since this is the first nonparametric significance test able to account for measurement error, it is difficult to give a direct comparison to any existing tests. However, we report results for the test of Delgado and González-Manteiga (2001) (DM henceforth) as well as a Wald test based on an IV regression with functional form: $\beta_{0}+\beta_{1} X_{1}+\beta_{2} X_{1}^{2}+\beta_{3} X_{2}+\beta_{4} X_{2}^{2}$; a repeated measurement, as well as its square, are used as the instruments.

The true, unobservable regressors $\left(X^{*}, Z^{*}\right)$ are each distributed as $N(0,1)$ with a correlation of 0.4. The contaminated regressors are given by $X=X^{*}+\epsilon$ and $Z=Z^{*}+\nu$. In order to fairly compare our test with the available alternatives, we do not assume we know $f_{\epsilon}^{\mathrm{ft}}$ or $f_{\nu}^{\mathrm{ft}} .{ }^{3}$ Instead, we assume a repeated measurement of each contaminated regressor is available, given by $X^{r}=X^{*}+\epsilon^{r}$ and $Z^{r}=Z^{*}+\nu^{r}$, where $\epsilon^{r}$ is distributed independently and identically to $\epsilon$ (as are $\nu^{r}$ and $\nu$ ). For the ordinary smooth case, both $\epsilon$ and $\nu$ are drawn independently from the Laplace distribution with a standard deviation of $1 / 3$, giving a signal to noise ratio of three. For the supersmooth case, we use a zero mean Gaussian error with standard deviation also equal to $1 / 3$. Since both distributions are symmetric around zero, we can use the repeated data to estimate the error characteristic functions as outlined in Section 5 .

The infinite-order flat-top kernel of McMurry and Politis (2004) is used throughout all simulations. This kernel is defined by its Fourier transform

$$
K^{\mathrm{ft}}(t)= \begin{cases}1 & \text { if }|t| \leq 0.05, \\ \exp \left\{\frac{-\exp \left(-(|t|-0.05)^{2}\right)}{(|t|-1)^{2}}\right\} & \text { if } 0.05<|t|<1 \\ 0 & \text { if }|t| \geq 1\end{cases}
$$

and satisfies Assumptions $\mathrm{O}$ (iii) and S (iii). Results are reported for two sample sizes $n=\{250,500\}$.

Unfortunately, there are currently no theoretical results on the optimal choice of bandwidth for testing in the presence of measurement error. It is beyond the scope of this paper to investigate such issues. However, we suggest using the procedure put forth by Bissantz et al. (2007) to select the optimal bandwidths for each individual deconvolution kernel estimator, i.e. for $\tilde{r}, \tilde{f}$, and $\tilde{h}$. The bandwidth selection procedure involves two steps. First, a pilot bandwidth is selected that is oversmoothing, denoted $b_{n}^{0}$. It is crucial that the initial bandwidth is larger than the optimal choice; however, beyond

\footnotetext{
${ }^{3}$ The results are slightly better when $f_{\epsilon}^{\mathrm{ft}}$ and $f_{\nu}^{\mathrm{ft}}$ are assumed known; however, the difference is small.
} 
this, the procedure is relatively insensitive to this initial selection. The plug-in bandwidth of Delaigle and Gijbels (2004) is used in these simulations, but, as suggested in Bissantz et al. (2007), this choice is multiplied by two to ensure it is oversmoothing. Second, a set of potential bandwidths is created, $b_{n, j}=b_{n}^{0}(j / J)$ for $j=1, \ldots, J$. Denote by $\tilde{f}_{j}$ the estimator $\tilde{f}$ which uses the $j$-th bandwidth in this set (an analogous procedure is used for $\tilde{r}$ and $\tilde{h}$ ). The selected bandwidth is the largest bandwidth $b_{n, j}$ such that $d_{j-1, j}^{(\infty)} \equiv\left\|\tilde{f}_{j-1}-\tilde{f}_{j}\right\|_{\infty}$ is larger than $\rho d_{J-1, J}^{(\infty)}$ for some $\rho>1$. To select $J$ and $\rho$, we follow the suggestion of Kato and Sasaki (2018) and choose $J=4 \log (n)$ and $\rho=0.4 \log (n)$.

For the test of DM, a rule-of-thumb bandwidth, $b_{\mathrm{dm}}=n^{-1 / 3}$, is used; this is taken from the simulations carried out in DM. Results are also reported for half of this value and double this value.

The critical values for our test are constructed using the bootstrap procedure outlined at the end of Section 5 with 499 replications. For DM, the bootstrap procedure denoted $C_{n}^{* *}$ in their paper is used. The perturbation random variable for their bootstrap is the Mammen two-point distribution. All results are based on 1000 Monte Carlo replications.

Table 1 shows results for the level accuracy of the three tests. The columns labelled 'CM' and 'KS' report the proportion of rejections for the Cramér-von Mises test and the Kolmogorov-Smirnov test proposed in this paper, respectively. The column labelled 'DM' refers to the test of Delgado and González-Manteiga (2001), and 'IV' displays results for the Wald test based on the IV quadratic regression. Tables 2, 3, and 4 display the power results for three different alternatives. Note that the power results are empirically adjusted to give a fair comparison of the three methods. ${ }^{4}$

Table 1: $Y=1+X+X^{2}+U$

\begin{tabular}{|c|c|c|c|c|c|c|c|c|c|c|c|c|c|}
\hline \multirow{3}{*}{$n$} & \multirow{3}{*}{ Level } & \multicolumn{6}{|c|}{ Ordinary Smooth } & \multicolumn{6}{|c|}{ Supersmooth } \\
\hline & & \multirow{2}{*}{$\mathrm{CM}$} & \multirow{2}{*}{$\mathrm{KS}$} & \multirow{2}{*}{ IV } & \multicolumn{3}{|c|}{ DM } & \multirow{2}{*}{$\mathrm{CM}$} & \multirow{2}{*}{$\mathrm{KS}$} & \multirow{2}{*}{ IV } & \multicolumn{3}{|c|}{$\mathrm{DM}$} \\
\hline & & & & & $0.5 b_{\mathrm{dm}}$ & $b_{\mathrm{dm}}$ & $2 b_{\mathrm{dm}}$ & & & & $0.5 b_{\mathrm{dm}}$ & $b_{\mathrm{dm}}$ & $2 b_{\mathrm{dm}}$ \\
\hline \multirow{2}{*}{250} & $5 \%$ & 6.0 & 6.7 & 8.1 & 15.1 & 14.5 & 25.1 & 6.7 & 6.7 & 10.1 & 15.3 & 18.8 & 24.9 \\
\hline & $10 \%$ & 13.2 & 13.5 & 12.1 & 25.4 & 26.0 & 42.3 & 13.1 & 13.4 & 15.1 & 25.2 & 30.1 & 39.7 \\
\hline \multirow{2}{*}{500} & $5 \%$ & 6.3 & 6.8 & 11.2 & 22.1 & 26.5 & 30.1 & 6.0 & 6.4 & 8.8 & 28.5 & 29.0 & 36.7 \\
\hline & $10 \%$ & 12.3 & 13.5 & 18.0 & 35.2 & 40.7 & 47.8 & 12.2 & 13.1 & 15.5 & 41.8 & 43.2 & 51.6 \\
\hline
\end{tabular}

\footnotetext{
${ }^{4}$ Empirically adjusted power results are obtained in the following way. Using the null model from Table 1, for each parameter setting and for each nominal level we first obtain the quantile at which the empirical rejection rate is equal to the nominal level, say the $\hat{Q}_{\alpha}^{t h}$ quantile for an $\alpha$ nominal level. In all subsequent models, the critical value is taken as the $\hat{Q}_{\alpha}^{t h}$ quantile from the bootstrap distribution, rather than the typical $(1-\alpha)$ quantile.
} 
Table 2: $Y=1+X+X^{2}+\frac{2}{\sqrt{n}} Z^{2}+U$

\begin{tabular}{|c|c|c|c|c|c|c|c|c|c|c|c|c|c|}
\hline \multirow{3}{*}{$n$} & \multirow{3}{*}{ Level } & \multicolumn{6}{|c|}{ Ordinary Smooth } & \multicolumn{6}{|c|}{ Supersmooth } \\
\hline & & \multirow{2}{*}{$\mathrm{CM}$} & \multirow{2}{*}{ KS } & \multirow{2}{*}{ IV } & \multicolumn{3}{|c|}{ DM } & \multirow{2}{*}{$\mathrm{CM}$} & \multirow{2}{*}{$\mathrm{KS}$} & \multirow{2}{*}{ IV } & \multicolumn{3}{|c|}{$\mathrm{DM}$} \\
\hline & & & & & $0.5 b_{\mathrm{dm}}$ & $b_{\mathrm{dm}}$ & $2 b_{\mathrm{dm}}$ & & & & $0.5 b_{\mathrm{dm}}$ & $b_{\mathrm{dm}}$ & $2 b_{\mathrm{dm}}$ \\
\hline \multirow{2}{*}{250} & $5 \%$ & 34.2 & 29.5 & 53.9 & 14.4 & 13.0 & 12.1 & 28.3 & 26.8 & 52.8 & 16.1 & 16.6 & 22.2 \\
\hline & $10 \%$ & 46.6 & 41.7 & 66.4 & 30.6 & 30.7 & 29.0 & 45.8 & 41.5 & 67.5 & 28.4 & 29.7 & 30.9 \\
\hline \multirow{2}{*}{500} & $5 \%$ & 30.3 & 23.9 & 44.3 & 14.2 & 16.9 & 18.4 & 32.4 & 28.5 & 50.5 & 18.7 & 20.1 & 23.9 \\
\hline & $10 \%$ & 47.6 & 35.9 & 58.7 & 28.6 & 29.0 & 30.2 & 52.2 & 41.3 & 66.2 & 28.8 & 29.9 & 34.3 \\
\hline
\end{tabular}

Table 3: $Y=1+X+X^{2}+\frac{2}{\sqrt{n}} \sin (Z)+U$

\begin{tabular}{|c|c|c|c|c|c|c|c|c|c|c|c|c|c|}
\hline \multirow{3}{*}{$n$} & \multirow{3}{*}{ Level } & \multicolumn{6}{|c|}{ Ordinary Smooth } & \multicolumn{6}{|c|}{ Supersmooth } \\
\hline & & \multirow{2}{*}{$\mathrm{CM}$} & \multirow{2}{*}{$\mathrm{KS}$} & \multirow{2}{*}{ IV } & \multicolumn{3}{|c|}{$\mathrm{DM}$} & \multirow{2}{*}{$\mathrm{CM}$} & \multirow{2}{*}{$\mathrm{KS}$} & \multirow{2}{*}{ IV } & \multicolumn{3}{|c|}{ DM } \\
\hline & & & & & $0.5 b_{\mathrm{dm}}$ & $b_{\mathrm{dm}}$ & $2 b_{\mathrm{dm}}$ & & & & $0.5 b_{\mathrm{dm}}$ & $b_{\mathrm{dm}}$ & $2 b_{\mathrm{dm}}$ \\
\hline \multirow{2}{*}{250} & $5 \%$ & 41.0 & 39.9 & 7.5 & 24.2 & 25.7 & 25.5 & 36.9 & 36.1 & 5.5 & 25.0 & 29.2 & 32.4 \\
\hline & $10 \%$ & 51.8 & 52.2 & 13.3 & 38.2 & 40.1 & 42.2 & 51.5 & 48.6 & 10.4 & 37.1 & 38.3 & 38.6 \\
\hline \multirow{2}{*}{500} & $5 \%$ & 32.8 & 37.5 & 6.7 & 23.2 & 26.7 & 27.7 & 39.1 & 41.8 & 6.2 & 30.0 & 32.5 & 33.4 \\
\hline & $10 \%$ & 46.2 & 49.4 & 11.2 & 39.8 & 40.2 & 40.4 & 52.7 & 52.4 & 10.5 & 40.0 & 41.6 & 43.8 \\
\hline
\end{tabular}

Table 4: $Y=1+X+X^{2}+\frac{5}{\sqrt{n}} X \sin (Z)+U$

\begin{tabular}{|c|c|c|c|c|c|c|c|c|c|c|c|c|c|}
\hline \multirow{3}{*}{$n$} & \multirow{3}{*}{ Level } & \multicolumn{6}{|c|}{ Ordinary Smooth } & \multicolumn{6}{|c|}{ Supersmooth } \\
\hline & & \multirow{2}{*}{$\mathrm{CM}$} & \multirow{2}{*}{ KS } & \multirow{2}{*}{ IV } & \multicolumn{3}{|c|}{$\mathrm{DM}$} & \multirow{2}{*}{$\mathrm{CM}$} & \multirow{2}{*}{$\mathrm{KS}$} & \multirow{2}{*}{ IV } & \multicolumn{3}{|c|}{$\mathrm{DM}$} \\
\hline & & & & & $0.5 b_{\mathrm{dm}}$ & $b_{\mathrm{dm}}$ & $2 b_{\mathrm{dm}}$ & & & & $0.5 b_{\mathrm{dm}}$ & $b_{\mathrm{dm}}$ & $2 b_{\mathrm{dm}}$ \\
\hline \multirow{2}{*}{250} & $5 \%$ & 34.6 & 35.6 & 6.3 & 20.8 & 22.5 & 20.5 & 30.1 & 30.9 & 5.0 & 20.4 & 25.5 & 26.8 \\
\hline & $10 \%$ & 43.8 & 46.2 & 13.0 & 35.6 & 38.0 & 38.7 & 44.5 & 43.3 & 11.8 & 33.6 & 34.3 & 32.4 \\
\hline \multirow{2}{*}{500} & $5 \%$ & 27.8 & 40.6 & 5.0 & 21.8 & 22.2 & 24.4 & 32.7 & 39.0 & 5.1 & 25.4 & 26.6 & 30.2 \\
\hline & $10 \%$ & 33.4 & 46.5 & 11.3 & 38.2 & 35.1 & 36.3 & 46.5 & 50.5 & 10.0 & 34.4 & 35.2 & 40.8 \\
\hline
\end{tabular}

The results are encouraging and appear to reflect the theoretical findings. The bootstrap procedure keeps the size of the Cramér-von Mises test and the Kolmogorov-Smirnov test reasonably close to the nominal value for both types of measurement error but with a general over-rejection. The Wald test based on the IV also shows an over-rejection in all cases which is slightly more severe than our tests. In contrast, the DM test displays severe size distortions which are exacerbated when using a larger 
bandwidth; the test appears to be almost unusable in some cases. However, we should keep in mind that this test is not designed to deal with situations of measurement error.

Table 2 displays power results for a quadratic alternative. In this case, the Wald test is conducted on a correctly specified model and acts as a benchmark with which to compare the other tests. As expected, the Wald test dominates the tests of this paper and that of DM. However, it is promising to see that, at least at the $10 \%$ level, the power of our tests do not fall far behind that of the Wald test, and in all cases dominate the test of DM. Given that the alternative is drifting at the rate $n^{-1 / 2}$, it is also encouraging to see that the power of our tests show no substantial decline - and even increase in some situations. Interestingly, there appears to be little difference in power in the ordinary smooth and supersmooth settings for our tests.

In Tables 3 and 4, a sinusoidal and an interactive sinusoidal alternative are considered, respectively. The results are broadly similar in each case. It seems that neither the Cramér-von Mises test nor the Kolmogorov-Smirnov test dominates the other; the Kolmogorov-Smirnov test is preferable with an interactive sinusoidal alternative, while the Cramér-von Mises test has better power against a quadratic alternative. The Wald test struggles to detect the higher nonlinearity in Tables 3 and 4 , reflecting the arguments given in Section 2, while our tests and the DM test perform well in these settings. However, in all cases, our tests show the highest power.

\section{Conclusion}

This paper develops, to the best of our knowledge, the first nonparametric significance test for regression models with classical measurement error in the regressors. The test is flexible in that the measurement error can enter the model through the regressors of interest, through the controlling variables, or through both. Furthermore, we allow for the characteristic function of the error distribution to be estimated. We derive the asymptotic properties of the test and show it can detect local alternatives at the $\sqrt{n}$-rate for both ordinary smooth and supersmooth measurement error. As a cost of this $\sqrt{n}$-rate, the asymptotic distribution is case dependent and difficult to estimate in practice. However, a consistent bootstrap procedure is provided to obtain critical values and is shown to work well in finite samples. Although a practical approach to the choice of bandwidth is given, the problem of choosing an optimal bandwidth for testing in the presence of measurement error is still an open issue that needs to be resolved. 


\section{References}

[1] Adusumilli, K., Kurisu, D., Otsu, T. and Whang, Y.J. (2019) Inference on distribution functions under measurement error. Journal of Econometrics, first published online 26 September 2019. doi.org/10.1016/j.jeconom.2019.09.002

[2] Ahn, H. and Powell, J.L. (1993) Semiparametric estimation of censored selection models with a nonparametric selection mechanism. Journal of Econometrics, 58(1-2), 3-29.

[3] Bierens, H.J. (1982) Consistent model specification tests. Journal of Econometrics, 20(1), 105-134.

[4] Bierens, H.J. (1990) A consistent conditional moment test of functional form. Econometrica, 1443-1458.

[5] Bierens, H.J. and Ploberger, W. (1997) Asymptotic theory of integrated conditional moment tests. Econometrica, 1129-1151.

[6] Bissantz, N., Dümbgen, L., Holzmann, H. and Munk, A. (2007) Non-parametric confidence bands in deconvolution density estimation. Journal of the Royal Statistical Society: Series B, 69(3), 483-506.

[7] Butucea, C. and Taupin, M.L. (2008) New M-estimators in semi-parametric regression with errors in variables. Annales de l'IHP Probabilités et Statistiques, 44(3), 393-421.

[8] Carroll, R.J. and Hall, P. (1988) Optimal rates of convergence for deconvolving a density. Journal of the American Statistical Association, 83(404), 1184-1186.

[9] Chen, X. and Fan, Y. (1999) Consistent hypothesis testing in semiparametric and nonparametric models for econometric time series. Journal of Econometrics 91(2), 373-401.

[10] Chen, X. and White, H. (1998) Central limit and functional central limit theorems for Hilbertvalued dependent heterogeneous arrays with applications. Econometric Theory, 14(2), 260-284.

[11] Chernozhukov, V., Chetverikov, D. and Kato, K. (2014) Gaussian approximation of suprema of empirical processes. Annals of Statistics, 42(4), 1564-1597. 
[12] Chernozhukov, V., Chetverikov, D. and Kato, K. (2015) Comparison and anti-concentration bounds for maxima of Gaussian random vectors. Probability Theory and Related Fields, 162(1-2), $47-70$.

[13] Chernozhukov, V. Chetverikov, D. and Kato, K. (2016) Empirical and multiplier bootstraps for suprema of empirical processes of increasing complexity, and related Gaussian couplings. Stochastic Processes and their Applications, 126(12), 3632-3651.

[14] Delaigle, A. and Hall, P. (2016) Methodology for non-parametric deconvolution when the error distribution is unknown. Journal of the Royal Statistical Society: Series B, 78(1), 231-252.

[15] Delaigle, A., Hall, P. and Meister, A. (2008) On deconvolution with repeated measurements. Annals of Statistics, 36(2), 665-685.

[16] Delgado, M.A. and González-Manteiga, W. (2001) Significance testing in nonparametric regression based on the bootstrap. Annals of Statistics, 29, 1469-1507.

[17] Dong, H., Otsu, T. and Taylor, L. (2019) Average derivative estimation under measurement error. Working paper.

[18] Dudley, R.M. (2002) Real Analysis and Probability. Cambridge University Press.

[19] Fan, J. and Truong, Y.K. (1993) Nonparametric regression with errors in variables. Annals of Statistics, 1900-1925.

[20] Fan, Y. (1995) Average derivative estimation with errors-in-variables. Journal of Nonparametric Statistics, 4(4), 395-407.

[21] Fisher, R. A. (1929) The statistical method in psychical research. Proceedings of the Society for Psychical Research, 39, 189-192.

[22] Giné, E. and Nickl, R., 2016. Mathematical Foundations of Infinite-Dimensional Statistical Models. Cambridge University Press.

[23] González-Manteiga, W. and Crujeiras, R.M. (2013) An updated review of goodness-of-fit tests for regression models. Test, 22(3), 361-411. 
[24] Hall, P. and Ma, Y. (2007a) Semiparametric estimators of functional measurement error models with unknown error. Journal of the Royal Statistical Society: Series B, 69(3), 429-446.

[25] Hall, P. and Ma, Y. (2007b) Testing the suitability of polynomial models in errors-in-variables problems. Annals of Statistics, 35(6), 2620-2638.

[26] Hall, P. and Meister, A. (2007) A ridge-parameter approach to deconvolution. Annals of Statistics, $35(4), 1535-1558$.

[27] Härdle, W. and Mammen, E. (1993) Comparing nonparametric versus parametric regression fits. Annals of Statistics, 1926-1947.

[28] Hu, Y. and Ridder, G. (2012) Estimation of nonlinear models with mismeasured regressors using marginal information. Journal of Applied Econometrics, 27(3), 347-385.

[29] Kato, K. and Sasaki, Y. (2018) Uniform confidence bands in deconvolution with unknown error distribution. Journal of Econometrics, 207(1), 129-161.

[30] Kato, K. and Sasaki, Y. (2019) Uniform confidence bands for nonparametric errors-in-variables regression. Journal of Econometrics, 213(2), 516-555.

[31] Kotlarski, I. (1967) On characterizing the gamma and the normal distribution. Pacific Journal of Mathematics, 20(1), 69-76.

[32] Lavergne, P. and Vuong, Q.H. (2000) Nonparametric significance testing. Econometric Theory, $16(4), 576-601$.

[33] Lavergne, P. and Vuong, Q.H. (1996) Nonparametric selection of regressors: The nonnested case. Econometrica, 207-219.

[34] Li, T. and Vuong, Q. (1998) Nonparametric estimation of the measurement error model using multiple indicators. Journal of Multivariate Analysis, 65(2), 139-165.

[35] Ma, Y. and Li, R. (2010) Variable selection in measurement error models. Bernoulli, 16(1), 274300.

[36] McMurry, T. L. and D. N. Politis (2004) Nonparametric regression with infinite order flat-top kernels, Journal of Nonparametric Statistics, 16, 549-562. 
[37] Meister, A. (2009) Deconvolution Problems in Nonparametric Statistics, Springer.

[38] Powell, J.L., Stock, J.H. and Stoker, T.M. (1989) Semiparametric estimation of index coefficients. Econometrica, 1403-1430.

[39] Robinson, P.M. (1989) Hypothesis testing in semiparametric and nonparametric models for econometric time series. The Review of Economic Studies, 56(4), 511-534.

[40] Schennach, S.M. (2016) Recent advances in the measurement error literature. Annual Review of Economics, 8, 341-377.

[41] Stefanski, L.A. and Carroll, R.J. (1990) Deconvolving kernel density estimators. Statistics, 21(2), 169-184.

[42] Stinchcombe, M.B. and White, H. (1998) Consistent specification testing with nuisance parameters present only under the alternative. Econometric Theory, 14(03), 295-325.

[43] Stute, W. (1997) Nonparametric model checks for regression. Annals of Statistics, 613-641.

[44] Su, L. and White, H. (2008) A nonparametric Hellinger metric test for conditional independence. Econometric Theory, 24(4), 829-864.

[45] Van Der Vaart, A. W. and Wellner, J. A. (1996) Weak Convergence and Empirical Processes, Springer.

[46] Van Es, A. J. and Uh H.-W. (2005) Asymptotic normality for kernel type deconvolution estimators, Scandinavian Journal of Statistics, 32, 467-483.

[47] Zhang, X., Wang, H., Ma, Y. and Carroll, R.J. (2017) Linear model selection when covariates contain errors. Journal of the American Statistical Association, 112(520), 1553-1561.

[48] Zhu, L. and Cui, H. (2003) A Semi-parametric regression model with errors in variables. Scandinavian Journal of Statistics, 30(2), 429-442. 


\section{A Proofs of Theorems}

\section{A.1 Proof of Theorem 1}

Define $d_{j}=\left(X_{j}, Y_{j}, Z_{j}\right)$ and write $T_{n}(\xi)=\frac{(n-1)}{n} U_{n}(\xi)$, where $U_{n}(\xi)=\left(\begin{array}{c}n \\ 2\end{array}\right)^{-1} \sum_{j=1}^{n-1} \sum_{k=j+1}^{n} p_{n}\left(d_{j}, d_{k} ; \xi\right)$ for $\xi=\left(\xi_{1}, \xi_{2}\right)$ is a second-order U-statistic with symmetric kernel

$$
p_{n}\left(d_{j}, d_{k} ; \xi\right)=\frac{1}{2 b_{n}^{3}} \iint\left\{\begin{array}{c}
\left(Y_{k}-Y_{j}\right) \mathbb{K}_{\epsilon}\left(\frac{x-X_{j}}{b_{n}}\right) \mathbb{K}_{\epsilon}\left(\frac{x-X_{k}}{b_{n}}\right) \mathbb{K}_{\nu}\left(\frac{z-Z_{k}}{b_{n}}\right) \\
+\left(Y_{j}-Y_{k}\right) \mathbb{K}_{\epsilon}\left(\frac{x-X_{k}}{b_{n}}\right) \mathbb{K}_{\epsilon}\left(\frac{x-X_{j}}{b_{n}}\right) \mathbb{K}_{\nu}\left(\frac{z-Z_{j}}{b_{n}}\right)
\end{array}\right\} e^{\mathrm{i}(x, z) \xi} d x d z
$$

Let $U_{n}^{*}(\xi)=E\left[r_{n}\left(d_{1} ; \xi\right)\right]+\frac{2}{n} \sum_{j=1}^{n}\left\{r_{n}\left(d_{j} ; \xi\right)-E\left[r_{n}\left(d_{j} ; \xi\right)\right]\right\}$ for $r_{n}\left(d_{j} ; \xi\right)=E\left[p_{n}\left(d_{j}, d_{k} ; \xi\right) \mid d_{j}\right]$. By the Hájek projection (see, for example, Ahn and Powell, 1993, Lemma A.3), if

$$
E\left[\sup _{\xi \in \Xi} p_{n}\left(d_{j}, d_{k} ; \xi\right)^{2}\right]=o(n)
$$

then

$$
\sup _{\xi \in \Xi}\left|U_{n}(\xi)-U_{n}^{*}(\xi)\right|=o_{p}\left(n^{-1 / 2}\right)
$$

To show (A.1), note that

$$
\begin{aligned}
& E\left[\sup _{\xi \in \Xi} p_{n}\left(d_{j}, d_{k} ; \xi\right)^{2}\right] \\
& \leq \frac{2}{b_{n}^{6}} E\left[\begin{array}{c}
\sup _{\xi \in \Xi}\left|\iint \mathbb{K}_{\epsilon}\left(\frac{x-X_{j}}{b_{n}}\right) \mathbb{K}_{\epsilon}\left(\frac{x-X_{k}}{b_{n}}\right) \mathbb{K}_{\nu}\left(\frac{z-Z_{k}}{b_{n}}\right) e^{\mathrm{i}(x, z) \xi} d x d z\right|^{2} \\
\times\left\{E\left[Y_{j}^{2} \mid X_{j}^{*}\right]+E\left[Y_{k}^{2} \mid X_{k}^{*}, Z_{k}^{*}\right]\right\}
\end{array}\right] \\
& =\frac{2}{b_{n}^{2}} E\left[\begin{array}{c}
\sup _{\xi \in \Xi}\left|\iint \mathbb{K}_{\epsilon}\left(\tilde{x}+\frac{X_{k}-X_{j}}{b_{n}}\right) \mathbb{K}_{\epsilon}(\tilde{x}) \mathbb{K}_{\nu}(\tilde{z}) e^{\mathrm{i}\left(X_{k}+b_{n} \tilde{x}, Z_{k}+b_{n} \tilde{z}\right) \xi} d \tilde{x} d \tilde{z}\right|^{2} \\
\times\left\{E\left[Y_{j}^{2} \mid X_{j}^{*}\right]+E\left[Y_{k}^{2} \mid X_{k}^{*}, Z_{k}^{*}\right]\right\}
\end{array}\right] \\
& \leq \frac{1}{2 \pi^{2} b_{n}^{2}} E\left[\begin{array}{c}
\left\{\int \frac{\left|K^{\mathrm{ft}}(t)\right|}{\left|f_{\epsilon}^{\mathrm{ft}}\left(t / b_{n}\right)\right|}\left(\sup _{\xi \in \Xi} \frac{\left|K^{\mathrm{ft}}\left(-t+b_{n} \xi_{1}\right)\right|\left|K^{\mathrm{ft}}\left(b_{n} \xi_{2}\right)\right|}{\left|f_{\epsilon}^{\mathrm{ft}}\left(-t / b_{n}+\xi_{1}\right)\right|\left|f_{\nu}^{\mathrm{ft}}\left(\xi_{2}\right)\right|}\right) d t\right\}^{2} \\
\times\left\{E\left[Y_{j}^{2} \mid X_{j}^{*}\right]+E\left[Y_{k}^{2} \mid X_{k}^{*}, Z_{k}^{*}\right]\right\}
\end{array}\right] \\
& =O\left(b_{n}^{-2}\left(\inf _{|t| \leq 2 b_{n}^{-1}}\left|f_{\epsilon}^{\mathrm{ft}}(t)\right|\right)^{-4}\right) \text {, }
\end{aligned}
$$

where the first step follows by Jensen's inequality, the law of iterated expectations, and the independence between $(\epsilon, \nu)$ and $Y$ as imposed in Assumption D (ii), the second step follows by the change of variables $\tilde{x}=\frac{x-X_{k}}{b_{n}}$ and $\tilde{z}=\frac{z-Z_{k}}{b_{n}}$, the third step uses Lemma 1, and the last step follows from 
$E\left[Y^{2}\right]<\infty$ as assumed in Assumption D (i), $\inf _{\xi \in \Xi}\left|f_{\nu}^{\mathrm{ft}}\left(\xi_{2}\right)\right|>\underline{c}$ as assumed in Assumption D (ii), and the fact that $K^{\mathrm{ft}}$ is supported on $[-1,1]$ and is bounded as assumed in Assumption $\mathrm{O}$ (iii). Then, (A.1) follows by (A.3) and Assumption O (ii) and (iv).

For the bias term of $U_{n}^{*}(\xi)$, under $\mathrm{H}_{0}$, we claim that it is asymptotically negligible, i.e.

$$
\sup _{\xi \in \Xi}\left|E\left[r_{n}\left(d_{1} ; \xi\right)\right]\right|=o\left(n^{-1 / 2}\right)
$$

To show (A.4), using Lemma 2, we write $E\left[r_{n}\left(d_{1} ; \xi\right)\right]$ as

$$
E\left[r_{n}\left(d_{1} ; \xi\right)\right]=\frac{1}{b_{n}^{3}} \iint\left\{\begin{array}{c}
E\left[K\left(\frac{x-X^{*}}{b_{n}}\right)\right] E\left[Y K\left(\frac{x-X^{*}}{b_{n}}\right) K\left(\frac{z-Z^{*}}{b_{n}}\right)\right] \\
-E\left[Y K\left(\frac{x-X^{*}}{b_{n}}\right)\right] E\left[K\left(\frac{x-X^{*}}{b_{n}}\right) K\left(\frac{z-Z^{*}}{b_{n}}\right)\right]
\end{array}\right\} e^{\mathrm{i}(x, z) \xi} d x d z
$$

Then, (A.4) follows by Lemma 3. In particular, $\sup _{\xi \in \Xi}\left|E\left[r_{n}\left(d_{1} ; \xi\right)\right]\right|=O\left(b_{n}^{p}\right)=o\left(n^{-1 / 2}\right)$ under Assumption O.

For the main term of $U_{n}^{*}(\xi)$, we decompose $2 r_{n}\left(d_{j} ; \xi\right)=r_{1, n}\left(d_{j} ; \xi\right)+r_{2, n}\left(d_{j} ; \xi\right)$, where

$$
\begin{aligned}
& r_{1, n}\left(d_{j} ; \xi\right)=\iiint\left\{\begin{array}{c}
\mathbb{K}_{\epsilon}(\tilde{x}) \mathbb{K}_{\nu}(\tilde{z}) K\left(x^{*}\right) e^{\mathrm{i}\left(X_{j}+b_{n} \tilde{x}, Z_{j}+b_{n} \tilde{z}\right) \xi} \\
\times\left\{Y_{j} f-g\right\}\left(X_{j}+b_{n}\left(\tilde{x}-x^{*}\right)\right)
\end{array}\right\} d x^{*} d \tilde{x} d \tilde{z}, \\
& r_{2, n}\left(d_{j} ; \xi\right)=\iiint \int\left\{\begin{array}{c}
\mathbb{K}_{\epsilon}(\tilde{x}) K\left(x^{*}\right) K\left(z^{*}\right) e^{\mathrm{i}\left(X_{j}+b_{n} \tilde{x}, z\right) \xi} \\
\times\left\{g_{X^{*}, Z^{*}}-Y_{j} f_{X^{*}, Z^{*}}\right\}\left(X_{j}+b_{n}\left(\tilde{x}-x^{*}\right), z-b_{n} z^{*}\right)
\end{array}\right\} d x^{*} d z^{*} d \tilde{x} d z,
\end{aligned}
$$

which are obtained using Lemma 2 and the change of variables $\tilde{x}=\frac{x-X_{j}}{b_{n}}$ and $\tilde{z}=\frac{z-Z_{j}}{b_{n}}$.

Since $f, g, f_{X^{*}, Z^{*}}$, and $g_{X^{*}, Z^{*}}$ are $p$-times differentiable with $p>1+2 \alpha$ under Assumption O, by expanding $\left\{Y_{j} f-g\right\}\left(X_{j}+b_{n}\left(\tilde{x}-x^{*}\right)\right)$ around $X_{j}$ to the $p^{\text {th }}$-order, we have $r_{1, n}\left(d_{j} ; \xi\right)=$ $r_{1, n}^{*}\left(d_{j} ; \xi\right)+t_{1, n}\left(d_{j} ; \xi\right)$, where

$$
\begin{aligned}
& r_{1, n}^{*}\left(d_{j} ; \xi\right)=\sum_{l=0}^{p} \frac{b_{n}^{l} K^{\mathrm{ft}}\left(b_{n} \xi_{2}\right)}{l ! f_{\nu}^{\mathrm{ft}}\left(\xi_{2}\right)} \int \mathbb{K}_{\epsilon}(\tilde{x}) \tilde{x}^{l} e^{\mathrm{i} b_{n} \tilde{x} \xi_{1}} d \tilde{x}\left\{Y_{j} f^{(l)}-g^{(l)}\right\}\left(X_{j}\right) e^{\mathrm{i}\left(X_{j}, Z_{j}\right) \xi}, \\
& t_{1, n}\left(d_{j} ; \xi\right)=\sum_{l=0}^{p} \frac{b_{n}^{p}(-1)^{p-l} K^{\mathrm{ft}}\left(b_{n} \xi_{2}\right)}{l !(p-l) ! f_{\nu}^{\mathrm{ft}}\left(\xi_{2}\right)} \iint\left\{\begin{array}{c}
\mathbb{K}_{\epsilon}(\tilde{x}) \tilde{x}^{l} e^{\mathrm{i} b_{n} \tilde{x} \xi_{1}} K\left(x^{*}\right) x^{* p-l} \\
\times\left[\begin{array}{c}
\left\{Y_{j} f^{(p)}-g^{(p)}\right\}\left(\bar{X}_{j}\right) \\
-\left\{Y_{j} f^{(p)}-g^{(p)}\right\}\left(X_{j}\right)
\end{array}\right] e^{\mathrm{i}\left(X_{j}, Z_{j}\right) \xi}
\end{array}\right\} d x^{*} d \tilde{x},
\end{aligned}
$$


for some $\bar{X}_{j}$ lying between $X_{j}$ and $X_{j}+b_{n}\left(\tilde{x}-x^{*}\right)$, which are obtained using the binomial theorem, Lemma 1, and the properties of the kernel function $K$ as assumed in Assumption O (iii).

For the main term $r_{1, n}^{*}\left(d_{j} ; \xi\right)$, we have

$$
\sup _{\xi \in \Xi}\left|\frac{2}{n} \sum_{j=1}^{n}\left\{r_{1, n}^{*}\left(d_{j} ; \xi\right)-r_{1, \infty}^{\mathrm{os}}\left(d_{j} ; \xi\right)-E\left[r_{1, n}^{*}\left(d_{j} ; \xi\right)-r_{1, \infty}^{\mathrm{os}}\left(d_{j} ; \xi\right)\right]\right\}\right|=o_{p}\left(n^{-1 / 2}\right)
$$

for $r_{1, \infty}^{\mathrm{os}}\left(d_{j} ; \xi\right)=\sum_{l=0}^{\alpha} c_{l}^{\mathrm{os}}\left(\xi_{1}\right)\left\{Y_{j} f^{(l)}-g^{(l)}\right\}\left(X_{j}\right) e^{\mathrm{i}\left(X_{j}, Z_{j}\right) \xi} f_{\nu}^{\mathrm{ft}-1}\left(\xi_{2}\right)$, which follows by

$$
\begin{aligned}
& \operatorname{Var}\left(\sup _{\xi \in \Xi}\left|\frac{1}{\sqrt{n}} \sum_{j=1}^{n}\left\{r_{1, n}^{*}\left(d_{j} ; \xi\right)-r_{1, \infty}^{\mathrm{os}}\left(d_{j} ; \xi\right)\right\}\right|\right) \leq E\left[\sup _{\xi \in \Xi}\left\{r_{1, n}^{*}\left(d_{j} ; \xi\right)-r_{1, \infty}^{\mathrm{os}}\left(d_{j} ; \xi\right)\right\}^{2}\right] \\
= & O\left(\sup _{\xi \in \Xi}\left|K^{\mathrm{ft}}\left(b_{n} \xi_{2}\right)-1\right|^{2} E\left[\sup _{\xi \in \Xi}\left|\sum_{l=1}^{\alpha} c_{l}^{\mathrm{os}}\left(\xi_{1}\right)\left\{Y f^{(l)}-g^{(l)}\right\}(X)\right|^{2}\right]+b_{n}^{2}\right)=o(1),
\end{aligned}
$$

where the first equality follows by Lemma 5 and $\inf _{\xi \in \Xi}\left|f_{\nu}^{\mathrm{ft}}\left(\xi_{2}\right)\right|>\underline{c}$ as assumed in Assumption D (ii), and the second equality follows by Assumption $\mathrm{O}(\mathrm{v})$, the compactness of $\Xi$, and $K^{\mathrm{ft}}\left(b_{n} \xi_{2}\right) \rightarrow 1$ as $n \rightarrow \infty$.

For the residual term $t_{1, n}\left(d_{j} ; \xi\right)$, we claim that it is negligible, i.e.

$$
\sup _{\xi \in \Xi}\left|\frac{2}{n} \sum_{j=1}^{n}\left\{t_{1, n}\left(d_{j} ; \xi\right)-E\left[t_{1, n}\left(d_{j} ; \xi\right)\right]\right\}\right|=o_{p}\left(n^{-1 / 2}\right)
$$

which follows by

$$
\begin{aligned}
& \operatorname{Var}\left(\sup _{\xi \in \Xi}\left|\frac{1}{\sqrt{n}} \sum_{j=1}^{n} t_{1, n}\left(d_{j} ; \xi\right)\right|\right) \leq E\left[\sup _{\xi \in \Xi} t_{1, n}\left(d_{j} ; \xi\right)^{2}\right] \\
&= O\left(b _ { n } ^ { 2 p } \operatorname { m a x } _ { 0 \leq l \leq p } E \left[\int \int \left\{\mathbb{K}_{\epsilon}(\tilde{x}) \tilde{x}^{l} K\left(x^{*}\right) x^{* \alpha+1-l} \mid\right.\right.\right. \\
&\left.\left.\left.\times\left|\left\{Y f^{(p)}-g^{(p)}\right\}(\bar{X})-\left\{Y f^{(p)}-g^{(p)}\right\}(X)\right|\right\} d x^{*} d \tilde{x}\right]^{2}\right) \\
&= O\left(b_{n}^{2 p+2} \max _{0 \leq l \leq p} E\left[\iint\left\{\begin{array}{c}
\left|\mathbb{K}_{\epsilon}(\tilde{x}) \tilde{x}^{l} K\left(x^{*}\right) x^{* p-l}\left(\tilde{x}-x^{*}\right)\right| \\
\times\left\{|Y|\left|m_{1, p}\right|+\left|m_{2, p}\right|\right\}(X)
\end{array}\right\} d x^{*} d \tilde{x}\right]^{2}\right) \\
&= O\left(b_{n}^{2 p+2} \max _{0 \leq l \leq p+1}\left(\int\left|\mathbb{K}_{\epsilon}(\tilde{x}) \tilde{x}^{l}\right| d \tilde{x}\right)^{2} E\left[\left\{Y^{2} m_{1, p}^{2}+m_{2, p}^{2}\right\}(X)\right]\right)=O\left(b_{n}^{2(p-\alpha)+2}\right)=o(1),
\end{aligned}
$$


where the first equality follows by $\inf _{\xi \in \Xi}\left|f_{\nu}^{\mathrm{ft}}\left(\xi_{2}\right)\right|>\underline{c}$ as assumed in Assumption D (ii) and the boundedness of $K^{\mathrm{ft}}$ as assumed in Assumption $\mathrm{O}$ (iii), the second equality follows by Assumption $\mathrm{O}$ (i), and the last equality follows by $\sup _{0 \leq l \leq p+1} \int\left|\mathbb{K}_{\epsilon}(u) u^{l}\right| d u=O\left(b_{n}^{-\alpha}\right)$, which is obtained using Lemma 4.

Then, (A.5) and (A.6) together imply

$$
\sup _{\xi \in \Xi}\left|\frac{2}{n} \sum_{j=1}^{n}\left\{r_{1, n}\left(d_{j} ; \xi\right)-r_{1, \infty}^{\mathrm{os}}\left(d_{j} ; \xi\right)-E\left[r_{1, n}\left(d_{j} ; \xi\right)-r_{1, \infty}^{\mathrm{os}}\left(d_{j} ; \xi\right)\right]\right\}\right|=o_{p}\left(n^{-1 / 2}\right)
$$

By a similar argument, for $r_{2, \infty}^{\mathrm{os}}\left(d_{j} ; \xi\right)=\sum_{l=0}^{\alpha} c_{l}^{\mathrm{os}}\left(\xi_{1}\right) \int\left\{g_{X^{*}, Z^{*}}^{(l, 0)}-Y_{j} f_{X^{*}, Z^{*}}^{(l, 0)}\right\}\left(X_{j}, z\right) e^{\mathrm{i}\left(X_{j}, z\right) \xi} d z$, we

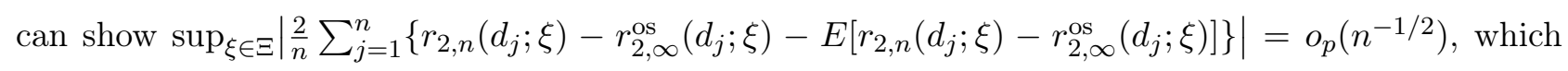
together with (A.7) imply

$$
\sup _{\xi \in \Xi}\left|\frac{2}{n} \sum_{j=1}^{n}\left\{r_{n}\left(d_{j} ; \xi\right)-r_{\infty}^{\mathrm{os}}\left(d_{j} ; \xi\right)-E\left[r_{n}\left(d_{j} ; \xi\right)-r_{\infty}^{\mathrm{os}}\left(d_{j} ; \xi\right)\right]\right\}\right|=o_{p}\left(n^{-1 / 2}\right)
$$

Note that Assumption $\mathrm{O}(\mathrm{v})$ implies $E\left[\int_{\Xi} r_{\infty}^{\mathrm{os}}\left(d_{j} ; \xi\right)^{2} d \xi\right]<\infty$. Then by Theorem 3.9 of Chen and White (1998), we have

$$
\frac{2}{\sqrt{n}} \sum_{j=1}^{n}\left\{r_{\infty}^{\mathrm{os}}\left(d_{j} ; \xi\right)-E\left[r_{\infty}^{\mathrm{os}}\left(d_{j} ; \xi\right)\right]\right\} \Rightarrow G^{\mathrm{os}}(\xi)
$$

The conclusion follows by combining (A.2), (A.4), (A.8), and (A.9).

\section{A.2 Proof of Theorem 2}

The proof follows along the same lines as Theorem 1, except that (A.1) follows by (A.3) (obtained using the boundedness of $K^{\mathrm{ft}}$ as assumed in Assumption S (iii) instead of Assumption $\mathrm{O}$ (iii)) and Assumption S (ii) and (iv). Also, $E\left[r_{n}\left(d_{1} ; \xi\right)\right]=0$ for any $\xi \in \Xi$ under Assumption S, i.e. the bias is completely removed by using the infinite-order kernel. To achieve this we must expand $\left\{Y_{j} f-\right.$ $g\}\left(X_{j}+b_{n}\left(\tilde{x}-x^{*}\right)\right)$ around $X_{j}$ to an infinite order instead of the $p^{\text {th }}$-order to adapt to the smoothness of the measurement error under Assumption S. In particular, by expanding $\left\{Y_{j} f-g\right\}\left(X_{j}+b_{n}\left(\tilde{x}-x^{*}\right)\right)$ around $X_{j}$ to an infinite order and using the binomial theorem, Lemma 1, and the properties of the 
kernel function $K$ as assumed in Assumption S (iii), we obtain

$$
r_{1, n}\left(d_{j} ; \xi\right)=\sum_{l=0}^{\infty} \frac{b_{n}^{l} K^{\mathrm{ft}}\left(b_{n} \xi_{2}\right)}{l ! f_{\nu}^{\mathrm{ft}}\left(\xi_{2}\right)} \int \mathbb{K}_{\epsilon}(\tilde{x}) \tilde{x}^{l} e^{\mathrm{i} b_{n} \tilde{x} \xi_{1}} d \tilde{x}\left\{Y_{j} f^{(l)}-g^{(l)}\right\}\left(X_{j}\right) e^{\mathrm{i}\left(X_{j}, Z_{j}\right) \xi}
$$

It is worthy to note that we do not have the residual term $t_{1, n}$ as in the ordinary smooth case because the expansion here is to the infinite order. For this $r_{1, n}\left(d_{j} ; \xi\right)$, we have

$$
\sup _{\xi \in \Xi}\left|\frac{2}{n} \sum_{j=1}^{n}\left\{r_{1, n}\left(d_{j} ; \xi\right)-r_{1, \infty}^{\mathrm{ss}}\left(d_{j} ; \xi\right)-E\left[r_{1, n}\left(d_{j} ; \xi\right)-r_{1, \infty}^{\mathrm{ss}}\left(d_{j} ; \xi\right)\right]\right\}\right|=o_{p}\left(n^{-1 / 2}\right),
$$

for $r_{1, \infty}^{\mathrm{ss}}\left(d_{j} ; \xi\right)=\sum_{l=0}^{\infty} c_{l}^{\mathrm{ss}}\left(\xi_{1}\right)\left\{Y_{j} f^{(l)}-g^{(l)}\right\}\left(X_{j}\right) e^{\mathrm{i}\left(X_{j}, Z_{j}\right) \xi} f_{\nu}^{\mathrm{ft}-1}\left(\xi_{2}\right)$, which follows by

$$
\begin{aligned}
& \operatorname{Var}\left(\sup _{\xi \in \Xi}\left|\frac{1}{\sqrt{n}} \sum_{j=1}^{n}\left\{r_{1, n}\left(d_{j} ; \xi\right)-r_{1, \infty}^{\mathrm{ss}}\left(d_{j} ; \xi\right)\right\}\right|\right) \leq E\left[\sup _{\xi \in \Xi}\left\{r_{1, n}\left(d_{j} ; \xi\right)-r_{1, \infty}^{\mathrm{ss}}\left(d_{j} ; \xi\right)\right\}^{2}\right] \\
= & O\left(\sup _{\xi \in \Xi}\left|K^{\mathrm{ft}}\left(b_{n} \xi_{2}\right)-1\right|^{2} E\left[\sup _{\xi \in \Xi}\left|\sum_{l=1}^{\infty} c_{l}^{\mathrm{ss}}\left(\xi_{1}\right)\left\{Y_{j} f^{(l)}-g^{(l)}\right\}\left(X_{j}\right)\right|^{2}\right]\right)=o(1),
\end{aligned}
$$

where the first equality follows by Lemma 6 and $\inf _{\xi \in \Xi}\left|f_{\nu}^{\mathrm{ft}}\left(\xi_{2}\right)\right|>\underline{c}$ as assumed in Assumption D (ii), and the last equality follows by Assumption $\mathrm{S}(\mathrm{v})$, the compactness of $\Xi$, and $K^{\mathrm{ft}}\left(b_{n} \xi_{2}\right) \rightarrow 1$ as $n \rightarrow \infty$.

By a similar argument, for $\left.r_{2, \infty}^{\mathrm{ss}}\left(d_{j} ; \xi\right)\right]=\sum_{l=0}^{\infty} c_{l}^{\mathrm{ss}}\left(\xi_{1}\right) \int\left\{g_{X^{*}, Z^{*}}^{(l, 0)}-Y_{j} f_{X^{*}, Z^{*}}^{(l, 0)}\right\}\left(X_{j}, z\right) e^{\mathrm{i}\left(X_{j}, z\right) \xi} d z$, we

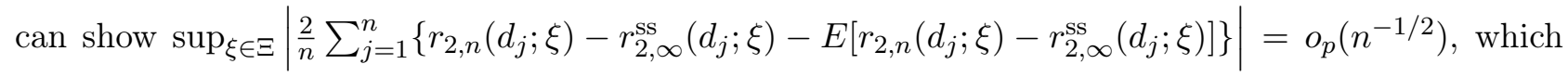
together with (A.10) imply

$$
\sup _{\xi \in \Xi}\left|\frac{2}{n} \sum_{j=1}^{n}\left\{r_{n}\left(d_{j} ; \xi\right)-r_{\infty}^{\mathrm{ss}}\left(d_{j} ; \xi\right)-E\left[r_{n}\left(d_{j} ; \xi\right)-r_{\infty}^{\mathrm{ss}}\left(d_{j} ; \xi\right)\right]\right\}\right|=o_{p}\left(n^{-1 / 2}\right) .
$$

Note that Assumption S (v) implies $E\left[\int_{\Xi} r_{\infty}^{\mathrm{ss}}\left(d_{j} ; \xi\right)^{2} d \xi\right]<\infty$. Then by Theorem 3.9 of Chen and White (1998), we have

$$
\frac{2}{\sqrt{n}} \sum_{j=1}^{n}\left\{r_{\infty}^{\mathrm{ss}}\left(d_{j} ; \xi\right)-E\left[r_{\infty}^{\mathrm{ss}}\left(d_{j} ; \xi\right)\right]\right\} \Rightarrow G^{\mathrm{ss}}(\xi)
$$

The conclusion follows by combining (A.2), (A.4), (A.11), and (A.12). 


\section{A.3 Proof of Theorem 3}

The proof follows along the same lines as Theorem 1 and 2, except that under $\mathrm{H}_{1 n}$, instead of (A.4), we have

$$
\sup _{\xi \in \Xi}\left|E\left[r_{n}\left(d_{1} ; \xi\right)\right]-n^{-1 / 2} \mu_{\Delta}(\xi)\right|=o\left(n^{-1 / 2}\right),
$$

which immediately follows by Lemma 3 and $\mathrm{H}_{1 n}$.

\section{A.4 Proof of Theorem 4}

We first introduce some notation needed in this proof. For some probability measure $Q$ on a measurable space $(S, \mathcal{S})$ and a class of measurable functions $\mathcal{F}$ on $S$ such that $\mathcal{F} \subset L^{2}(Q)$, let $N\left(\mathcal{F},|\cdot|_{Q, 2}, \delta\right)$ denote the $\delta$-covering number for $\mathcal{F}$ with respect to the $L^{2}(Q)$-seminorm $|\cdot|_{Q, 2}$. An envelope function $F: S \rightarrow[0, \infty]$ for $\mathcal{F}$ is characterised by the following property $F(u) \geq \sup _{f \in \mathcal{F}}|f(u)|$ for all $u \in S$ (van der Vaart and Wellner, 1996).

Since the proof for the ordinary smooth and supersmooth cases are very similar (given the results from Theorems 1 and 2), we provide the proof only for the ordinary smooth case. Define

$$
\bar{r}_{n}(\xi)=\frac{2}{\sqrt{n}} \sum_{j=1}^{n}\left\{r_{n}\left(d_{j} ; \xi\right)-E\left[r_{n}\left(d_{j} ; \xi\right)\right]\right\},
$$

for $r_{n}\left(d_{j} ; \xi\right)$ defined as in the proof of Theorem 1. Note that $\sup _{\xi \in \Xi}\left|\sqrt{n}\left\{T_{n}(\xi)-E\left[T_{n}(\xi)\right]\right\}-\bar{r}_{n}(\xi)\right|=$ $o_{p}\left(n^{-1 / 2}\right)$ from the proof of Theorem 1. Define the class of functions $\mathcal{F}_{n}^{(r)}=\left\{(x, y, z) \mapsto \bar{r}_{n}(x, y, z ; \xi)\right.$ : $\xi \in \Xi\}$. For $f \in \mathcal{F}_{n}^{(r)}$, consider the empirical process indexed by $\mathcal{F}_{n}^{(r)}: v_{n}(f)=\frac{1}{\sqrt{n}} \sum_{j=1}^{n} f\left(X_{j}, Y_{j}, Z_{j}\right)$. We first use Chernozhukov, Chetverikov and Kato (2016, Theorem 2.1) to show $\sup _{f \in \mathcal{F}_{n}^{(r)}}\left|v_{n}(f)\right|$ can be approximated by the supremum of a tight Gaussian process, $G_{n}(f)$, with zero mean and the same covariance function as $v_{n}(f)$. Thus, we begin by checking the required conditions for this approximation result.

As shown in the proof of Theorem 1, $\sup _{f \in \mathcal{F}_{n}^{(r)}}|f(x, y, z)|=O_{p}(1)$. Define $\sup _{f \in \mathcal{F}_{n}^{(r)}}|f(x, y, z)|=$ $C_{1} h(x, y, z)$ where $C_{1}<\infty$ and $h(x, y, z)=O_{p}(1)$ and an envelope function for $\mathcal{F}_{n}^{(r)}$ as $F_{n}^{(r)}(x, y, z)=$ $C_{2} h(x, y, z)$ where $C_{1}<C_{2}<\infty$. With this envelope function, Lemma 7 gives

$$
\sup _{Q} N\left(\mathcal{F}_{n}^{(r)},|\cdot|_{Q, 2}, \delta\right)=O_{p}(1)
$$


Using this result and the bound on the envelope function $F_{n}^{(r)}$, there exists a tight Gaussian process, $G_{n}(f)$, on $l^{\infty}\left(\mathcal{F}_{n}^{(r)}\right)$ with mean zero and the same covariance function as $\left\{v_{n}(f): f \in \mathcal{F}_{n}^{(r)}\right\}$. Following Kato and Sasaki (2019, Theorem 3.1), extend $v_{n}$ linearly to $\mathcal{F}_{n}^{(r)} \cup\left(-\mathcal{F}_{n}^{(r)}\right)=\left\{f,-f: f \in \mathcal{F}_{n}^{(r)}\right\}$ so that $\left|v_{n}\right|_{\mathcal{F}_{n}^{(r)}}=\sup _{f \in \mathcal{F}_{n}^{(r)} \cup\left(-\mathcal{F}_{n}^{(r)}\right)} v_{n}(f)$. Using Giné and Nickl (2016, Theorem 3.7.28), it can be shown that $G_{n}$ has uniformly continuous paths on the symmetric convex hull of $\mathcal{F}_{n}^{(r)}$ for the $L^{2}$ pseudo-metric and $\left\{G_{n}(f): f \in \mathcal{F}_{n}^{(r)} \cup\left(-\mathcal{F}_{n}^{(r)}\right)\right\}$ is a tight Gaussian process in $l^{\infty}\left(\mathcal{F}_{n}^{(r)} \cup\left(-\mathcal{F}_{n}^{(r)}\right)\right)$ with zero mean and the same covariance function as $\left\{v_{n}(f): f \in \mathcal{F}_{n}^{(r)} \cup\left(-\mathcal{F}_{n}^{(r)}\right)\right\}$.

The proof of Theorem 1 shows that $\sup _{\xi \in \Xi}\left|\bar{r}_{n}\left(d_{j} ; \xi\right)-\frac{2}{\sqrt{n}} \sum_{j=1}^{n}\left\{r_{\infty}^{\text {os }}\left(d_{j} ; \xi\right)-E\left[r_{\infty}^{\text {os }}\left(d_{j} ; \xi\right)\right]\right\}\right|=o_{p}(1)$ and by Assumption $4, \sup _{\xi \in \Xi} E\left|r_{\infty}^{\text {os }}(X, Y, Z ; \xi)\right|^{2+l}=O_{p}(1)$, thus, $\sup _{f \in \mathcal{F}_{n}^{(r)}} E|f(X, Y, Z ; \xi)|^{2+l}=$ $O_{p}(1)$ for $l=0,1,2$. Hence, Chernozhukov et al. (2016, Theorem 2.1) can be applied to $\mathcal{F}_{n}^{(r)} \cup\left(-\mathcal{F}_{n}^{(r)}\right)$ with, in the notation of that paper, $B(f)=0, q=4, A=O(1), V=O(1), b=O(1), \sigma=O(1)$, and $\gamma=O(1 / \log (n))$ to show that a random variable $V_{n}$ with the same distribution as $\sup _{f \in \mathcal{F}_{n}^{(r)}}\left|G_{n}(f)\right|$ exists and

$$
\left|\sup _{f \in \mathcal{F}_{n}^{(r)}}\right| v_{n}\left|-V_{n}\right|=O_{p}\left(\frac{\log (n)^{5 / 4}}{n^{1 / 4}}+\frac{\log (n)}{n^{1 / 6}}\right)=o_{p}(1) .
$$

Define $\bar{r}_{n}^{G}(\xi)=G_{n}\left(\bar{r}_{n}(\xi)\right)$ for $\xi \in \Xi$ so that $\bar{r}_{n}^{G}(\xi)$ is a tight Gaussian process in $l^{\infty}(\Xi)$ with zero mean and the same covariance function as $\bar{r}_{n}(\xi)$, giving $\sup _{\xi \in \Xi}\left|\bar{r}_{n}^{G}(\xi)\right|$ the same distribution as $V_{n}$. Now $\left|\sup _{\xi \in \Xi}\right| \sqrt{n}\left\{T_{n}(\xi)-E\left[T_{n}(\xi)\right]\right\}\left|-V_{n}\right| \leq\left|\sup _{\xi \in \Xi}\right| \sqrt{n}\left\{T_{n}(\xi)-E\left[T_{n}(\xi)\right]\right\}\left|-\sup _{\xi \in \Xi}\right| \bar{r}_{n}(\xi)|+| \sup _{\xi \in \Xi}\left|\bar{r}_{n}(\xi)\right|-V_{n} \mid=o_{p}(1)$, which implies $P\left\{\left|\sup _{\xi \in \Xi}\right| \sqrt{n}\left\{T_{n}(\xi)-E\left[T_{n}(\xi)\right]\right\}\left|-V_{n}\right|>\epsilon_{n}\right\} \leq \epsilon_{n}$ for some $\epsilon_{n}=O\left(n^{-c}\right)$ with $c>0$ (Dudley, 2002, Theorem 9.2.2). Combining these results, we have

$$
\begin{aligned}
& P\left\{\sup _{\xi \in \Xi}\left|\sqrt{n}\left\{T_{n}(\xi)-E\left[T_{n}(\xi)\right]\right\}\right| \leq w\right\} \geq P\left\{\sup _{\xi \in \Xi}\left|\bar{r}_{n}^{G}(\xi)\right| \leq w-\epsilon_{n}\right\}-\epsilon_{n} \\
\geq & P\left\{\sup _{\xi \in \Xi}\left|\bar{r}_{n}^{G}(\xi)\right| \leq w\right\}-4 \epsilon_{n}\left(1+E\left[\sup _{\xi \in \Xi}\left|\bar{r}_{n}^{G}(\xi)\right|\right]\right)
\end{aligned}
$$

where the second inequality follows from Chernozhukov, Chetverikov and Kato (2015, Theorem 3). Thus, it remains to bound $E\left[\sup _{\xi \in \Xi}\left|\bar{r}_{n}^{G}(\xi)\right|\right]$. In our case, Dudley's entropy integral bound gives

$$
E\left[\sup _{\xi \in \Xi}\left|\bar{r}_{n}^{G}(\xi)\right|\right]=O\left(\int_{0}^{1} \sqrt{\sup _{Q} N\left(\mathcal{F}_{n}^{(r)},|\cdot|_{Q, 2}, \delta\right)} d \delta\right)=O(1)
$$


where the second equality uses Lemma 7 . Therefore,

$$
P\left\{\sup _{\xi \in \Xi}\left|\sqrt{n}\left\{T_{n}(\xi)-E\left[T_{n}(\xi)\right]\right\}\right| \leq w\right\} \geq P\left\{\sup _{\xi \in \Xi}\left|\bar{r}_{n}^{G}(\xi)\right| \leq w\right\}-o(1) .
$$

The final two steps of the proof are to show

$$
\begin{gathered}
P\left\{\sup _{\xi \in \Xi}\left|\bar{r}_{n}^{\#}(\xi)\right| \leq w \mid d_{n}\right\} \geq P\left\{\sup _{\xi \in \Xi}\left|\sqrt{n}\left\{T_{n}^{\#}(\xi)-E\left[T_{n}^{\#}(\xi)\right]\right\}\right| \leq w \mid d_{n}\right\}-o(1), \\
P\left\{\sup _{\xi \in \Xi}\left|\bar{r}_{n}^{G}(\xi)\right| \leq w\right\} \geq P\left\{\sup _{\xi \in \Xi}\left|\bar{r}_{n}^{\#}(\xi)\right| \leq w \mid d_{n}\right\}-o(1),
\end{gathered}
$$

where $\bar{r}_{n}^{\#}(\xi)=\bar{r}_{n}\left(X^{\#}, Y^{\#}, Z^{\#} ; \xi\right)$ and $d_{n}=\left\{X_{j}, Y_{j}, Z_{j}\right\}_{j=1}^{n}$.

(A.14) can be shown by a straightforward application of the arguments of Theorem 1. For (A.15), we appeal to Chernozhukov et al. (2016, Theorem 2.3). To this end, define $v_{n}^{\#}(f)=$ $\frac{1}{\sqrt{n}} \sum_{k=1}^{n} f\left(X_{k}^{\#}, Y_{k}^{\#}, Z_{k}^{\#}\right)$, where $f \in \mathcal{F}_{n}^{(r)}$. Taking $B(f)=0, q=4, A=O(1), V=O(1), b=O(1)$, $\sigma=O(1)$, and $\gamma=O(1 / \log (n))$, Chernozhukov et al. (2016, Theorem 2.3) gives that there exists a random variable $V_{n}^{\#}$ with the same distribution (conditional on $d_{j}$ ) as $\sup _{f \in \mathcal{F}_{n}^{(r)}}\left|G_{n}(f)\right|$ and

$$
\left|\sup _{f \in \mathcal{F}_{n}^{(r)}}\right| v_{n}^{\#}(f)\left|-V_{n}^{\#}\right|=O_{p}\left(\frac{\log (n)^{9 / 4}}{n^{1 / 4}}+\frac{\log (n)^{2}}{n^{1 / 4}}+\frac{\log (n)}{n^{1 / 6}}\right)=o_{p}(1) .
$$

Therefore, for some $\epsilon_{n}=O\left(n^{-c}\right)$ for $c>0$,

$$
P\left\{\sup _{\xi \in \Xi}\left|\bar{r}_{n}^{\#}(\xi)\right| \leq w \mid d_{n}\right\} \geq P\left\{\sup _{\xi \in \Xi}\left|\bar{r}_{n}^{G}(\xi)\right| \leq w-\epsilon_{n}\right\}-\epsilon_{n} \geq P\left\{\sup _{\xi \in \Xi}\left|\bar{r}_{n}^{G}(\xi)\right| \leq w\right\}-o(1),
$$

where the second inequality follows again from Chernozhukov et al. (2015, Theorem 3) and Dudley's entropy integral bound. Combining these results, we have

$$
\begin{aligned}
& P\left\{\sup _{\xi \in \Xi}\left|\sqrt{n}\left\{T_{n}(\xi)-E\left[T_{n}(\xi)\right]\right\}\right| \leq w\right\} \geq P\left\{\sup _{\xi \in \Xi}\left|\bar{r}_{n}^{G}(\xi)\right| \leq w\right\}-o(1) \\
\geq & P\left\{\sup _{\xi \in \Xi}\left|\bar{r}_{n}^{\#}(\xi)\right| \leq w \mid d_{n}\right\}-o(1) \geq P\left\{\sup _{\xi \in \Xi}\left|\sqrt{n}\left\{T_{n}^{\#}(\xi)-E\left[T_{n}^{\#}(\xi)\right]\right\}\right| \leq w \mid d_{n}\right\}-o(1),
\end{aligned}
$$

and the conclusion follows by taking $w$ to be $\hat{c}_{\alpha}^{K S}$, i.e. the $(1-\alpha)^{\text {th }}$ quantile of $K S_{n}^{\#}$. 


\section{A.5 Proof of Theorem 5}

Let $\hat{\zeta}_{\epsilon}(t)=\frac{1}{n} \sum_{l=1}^{n} \zeta_{\epsilon, l}(t)$ and $\hat{\zeta}_{\nu}(t)=\frac{1}{n} \sum_{l=1}^{n} \zeta_{\nu, l}(t)$ for $\zeta_{\epsilon, l}(t)=\cos \left(t\left(X_{l}-X_{l}^{r}\right)\right)$ and $\zeta_{\nu, l}(t)=\cos \left(t\left(Z_{l}-\right.\right.$ $\left.Z_{l}^{r}\right)$ ), and $\zeta_{\epsilon}(t)=E\left[\zeta_{\epsilon, 1}(t)\right]$ and $\zeta_{\nu}(t)=E\left[\zeta_{\nu, 1}(t)\right]$. Note that $\hat{f}_{\epsilon}^{\mathrm{ft}}(t)=\left|\hat{\zeta}_{\epsilon}(t)\right|^{1 / 2}, \hat{f}_{\nu}^{\mathrm{ft}}(t)=\left|\hat{\zeta}_{\nu}(t)\right|^{1 / 2}$, $f_{\epsilon}^{\mathrm{ft}}(t)=\left|\zeta_{\epsilon}(t)\right|^{1 / 2}$, and $f_{\nu}^{\mathrm{ft}}(t)=\left|\zeta_{\nu}(t)\right|^{1 / 2}$.

For a random error $\eta \in\{\epsilon, \nu\}$, define

$$
\begin{aligned}
& A_{\eta}(u)=\frac{1}{2 \pi} \int e^{-\mathrm{i} t u} \frac{K^{\mathrm{ft}}(t)}{f_{\eta}^{\mathrm{ft}}\left(t / b_{n}\right)} \hat{\Pi}_{\eta}\left(t / b_{n}\right) d t, \\
& R_{\eta}(u)=\frac{1}{2 \pi} \int e^{-\mathrm{i} t u} \frac{K^{\mathrm{ft}}(t)}{f_{\eta}^{\mathrm{ft}}\left(t / b_{n}\right)} \hat{\Pi}_{\eta}^{\mathrm{res}}\left(t / b_{n}\right) d t,
\end{aligned}
$$

where $\hat{\Pi}_{\eta}(t)=\frac{1}{n} \sum_{l=1}^{n} \Pi_{\eta, l}(t)$ for $\Pi_{\eta, l}(t)=\frac{\zeta_{\eta}(t)-\zeta_{\eta, l}(t)}{2 \zeta_{\eta}(t)}$ and

$$
\hat{\Pi}_{\eta}^{\mathrm{res}}(t)=\frac{\left(2\left|\hat{\zeta}_{\eta}(t)\right|^{1 / 2}+\left|\zeta_{\eta}(t)\right|^{1 / 2}\right)\left(\left|\zeta_{\eta}(t)\right|^{1 / 2}-\left|\hat{\zeta}_{\eta}(t)\right|^{1 / 2}\right)^{2}}{\left|\zeta_{\eta}(t)\right|^{3 / 2}\left|\hat{\zeta}_{\eta}(t)\right|^{1 / 2}} .
$$

By expanding $\hat{\zeta}_{\eta}\left(t / b_{n}\right)$ around $\zeta_{\eta}\left(t / b_{n}\right)$, we obtain

$$
\hat{\mathbb{K}}_{\eta}(u)=\mathbb{K}_{\eta}(u)+A_{\eta}(u)+R_{\eta}(u) .
$$

Here $A_{\eta}(u)$ is the Fréchet derivative of $\hat{\mathbb{K}}_{\eta}(u)$ as a functional of $\hat{\zeta}_{\eta}\left(t / b_{n}\right)$ at $\zeta_{\eta}\left(t / b_{n}\right)$. Also note that $\hat{\Pi}_{\eta}(t)$ is of order $\left(\left|\zeta_{\eta}(t)\right|^{1 / 2}-\left|\hat{\zeta}_{\eta}(t)\right|^{1 / 2}\right)$ and $\hat{\Pi}_{\eta}^{\text {res }}(t)$ is of order $\left(\left|\zeta_{\eta}(t)\right|^{1 / 2}-\left|\hat{\zeta}_{\eta}(t)\right|^{1 / 2}\right)^{2}$. So, $R_{\eta}(u)$ is a higher order term than $A_{\eta}(u)$, and should be dominated by $A_{\eta}(u)$ asymptotically.

Decompose

$$
\hat{T}_{n}(\xi)=\hat{S}_{n}(\xi)+T_{1, n}(\xi)+\cdots+T_{4, n}(\xi)
$$

where

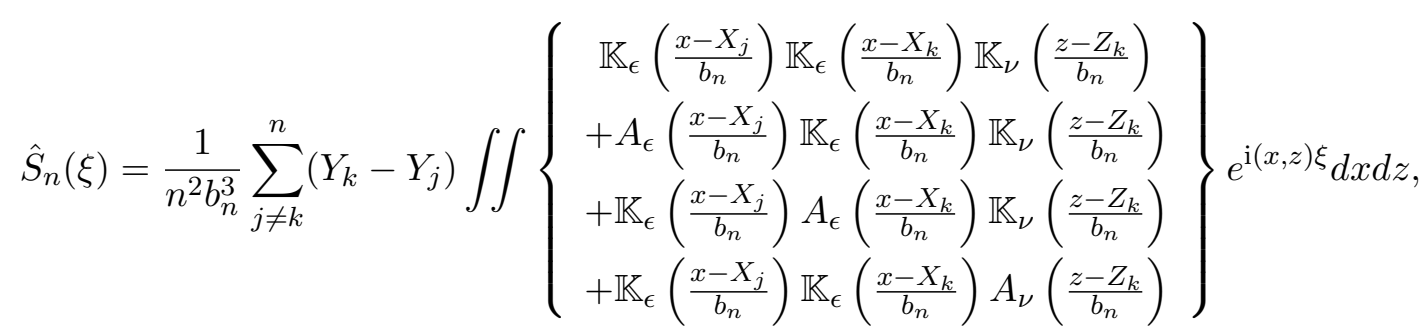




$$
\begin{aligned}
& T_{1, n}(\xi)=\frac{1}{n^{2} b_{n}^{3}} \sum_{j \neq k}^{n}\left(Y_{k}-Y_{j}\right) \iint \mathbb{K}_{\epsilon}\left(\frac{x-X_{j}}{b_{n}}\right) \mathbb{K}_{\epsilon}\left(\frac{x-X_{k}}{b_{n}}\right) R_{\nu}\left(\frac{z-Z_{k}}{b_{n}}\right) e^{\mathrm{i}(x, z) \xi} d x d z, \\
& T_{2, n}(\xi)=\frac{1}{n^{2} b_{n}^{3}} \sum_{j \neq k}^{n}\left(Y_{k}-Y_{j}\right) \iint\left\{\begin{array}{c}
A_{\epsilon}\left(\frac{x-X_{j}}{b_{n}}\right) \mathbb{K}_{\epsilon}\left(\frac{x-X_{k}}{b_{n}}\right) \\
+\mathbb{K}_{\epsilon}\left(\frac{x-X_{j}}{b_{n}}\right) A_{\epsilon}\left(\frac{x-X_{k}}{b_{n}}\right)
\end{array}\right\}\left\{\begin{array}{c}
A_{\nu}\left(\frac{z-Z_{k}}{b_{n}}\right) \\
+R_{\nu}\left(\frac{z-Z_{k}}{b_{n}}\right)
\end{array}\right\} e^{\mathrm{i}(x, z) \xi} d x d z, \\
& T_{3, n}(\xi)=\frac{1}{n^{2} b_{n}^{3}} \sum_{j \neq k}^{n}\left(Y_{k}-Y_{j}\right) \iint\left\{\begin{array}{c}
R_{\epsilon}\left(\frac{x-X_{j}}{b_{n}}\right) \mathbb{K}_{\epsilon}\left(\frac{x-X_{k}}{b_{n}}\right) \\
+\mathbb{K}_{\epsilon}\left(\frac{x-X_{j}}{b_{n}}\right) R_{\epsilon}\left(\frac{x-X_{k}}{b_{n}}\right)
\end{array}\right\} \hat{\mathbb{K}}_{\nu}\left(\frac{z-Z_{k}}{b_{n}}\right) e^{\mathrm{i}(x, z) \xi} d x d z, \\
& T_{4, n}(\xi)=\frac{1}{n^{2} b_{n}^{3}} \sum_{j \neq k}^{n}\left(Y_{k}-Y_{j}\right) \iint\left\{\begin{array}{c}
A_{\epsilon}\left(\frac{x-X_{j}}{b_{n}}\right) \\
+R_{\epsilon}\left(\frac{x-X_{j}}{b_{n}}\right)
\end{array}\right\}\left\{\begin{array}{c}
A_{\epsilon}\left(\frac{x-X_{k}}{b_{n}}\right) \\
+R_{\epsilon}\left(\frac{x-X_{k}}{b_{n}}\right)
\end{array}\right\} \hat{\mathbb{K}}_{\nu}\left(\frac{z-Z_{k}}{b_{n}}\right) e^{\mathrm{i}(x, z) \xi} d x d z,
\end{aligned}
$$

First, we show $T_{1, n}(\xi), \ldots, T_{4, n}(\xi)$ are asymptotic negligible, that is,

$$
\sup _{\xi \in \Xi}\left|T_{1, n}(\xi)\right|, \ldots, \sup _{\xi \in \Xi}\left|T_{4, n}(\xi)\right|=o_{p}\left(n^{-1 / 2}\right)
$$

For $T_{1, n}(\xi)$, we have

$$
\begin{aligned}
& T_{1, n}(\xi)=\frac{1}{2 \pi n^{2} b_{n}} \sum_{j \neq k}^{n}\left(Y_{k}-Y_{j}\right)\left\{\begin{array}{l}
\iint\left\{\begin{array}{c}
\frac{1}{2 \pi} \int e^{-\mathrm{i}\left(t_{1}+t_{2}-b_{n} \xi_{1}\right) \tilde{x}} d \tilde{x} \\
\times \frac{K^{\mathrm{ft}}\left(t_{1}\right) K^{\mathrm{ft}}\left(t_{2}\right) e^{\mathrm{i}\left(\frac{t_{1} X_{j}+t_{2} X_{k}}{b_{n}}\right)}}{f_{\epsilon}^{\mathrm{ft}}\left(t_{1} / b_{n}\right) f_{\epsilon}^{\mathrm{ft}}\left(t_{2} / b_{n}\right)}
\end{array}\right\} d t_{1} d t_{2} \\
\times \int\left\{\begin{array}{c}
\frac{1}{2 \pi} \int e^{-\mathrm{i}\left(t_{3}-b_{n} \xi_{2}\right) \tilde{z}} d \tilde{z} \\
\times \frac{K^{\mathrm{ft}}\left(t_{3}\right) e^{\mathrm{i} t_{3} z_{k} / b_{n}}}{f_{\nu}^{\mathrm{ft}}\left(t_{3} / b_{n}\right)} \hat{\Pi}_{\nu}^{\mathrm{res}}\left(t_{3} / b_{n}\right)
\end{array}\right\} d t_{3}
\end{array}\right\} \\
& =\frac{1}{2 \pi n^{2} b_{n}} \sum_{j \neq k}^{n}\left(Y_{k}-Y_{j}\right)\left\{\begin{array}{c}
\int \frac{K^{\mathrm{ft}}(t) K_{\mathrm{ft}}^{\mathrm{ft}}\left(-t+b_{n} \xi_{1}\right) e^{\mathrm{i}}}{f_{\epsilon}^{\mathrm{ft}}\left(t / b_{n}\right) f_{\epsilon}^{\mathrm{ft}}\left(-t / b_{n}+\xi_{1}\right)} \\
\times \frac{K^{\mathrm{ft}}\left(b_{n} \xi_{2}\right) e^{\mathrm{i}\left(X_{k}, Z_{k}\right) \xi}}{f_{\nu}^{\mathrm{ft}}\left(\xi_{2}\right)} \hat{\Pi}_{\nu}^{\mathrm{res}}\left(\xi_{2}\right)
\end{array}\right\} \\
& =O_{p}\left(\frac{1}{n b_{n}\left(\inf _{|t| \leq 2 b_{n}^{-1}}\left|f_{\epsilon}^{\mathrm{ft}}(t)\right|\right)^{2}}\right) \text {, }
\end{aligned}
$$

where the second equality follows by $\int \delta(u-b) m(u) d u=m(b)$ with the Dirac delta function $\delta(u)=$ $\frac{1}{2 \pi} \int e^{-\mathrm{i} u t} d t$, and the last equality follows from the properties of the kernel function $K$ as assumed in Assumption O (iii) and (B.4). By similar arguments, we can show $T_{2, n}(\xi)=O_{p}\left(\frac{\sup _{|t| \leq 2 b_{n}^{-1}}\left|\hat{\Pi}_{\epsilon}(t)\right|}{n^{1 / 2} b_{n}\left(\inf _{|t| \leq 2 b_{n}^{-1}}\left|f_{\epsilon}^{\mathrm{ft}}(t)\right|\right)^{2}}\right)$, $T_{3, n}(\xi)=O_{p}\left(\frac{\sup _{|t| \leq 2 b_{n}^{-1}}\left|\hat{\Pi}_{\epsilon}^{\mathrm{res}}(t)\right|}{b_{n}\left(\inf _{|t| \leq 2 b_{n}^{-1}}\left|f_{\epsilon}^{\mathrm{ft}}(t)\right|\right)^{2}}\right)$, and $T_{4, n}(\xi)=O_{p}\left(\frac{\sup _{|t| \leq 2 b_{n}^{-1}}\left|\hat{\Pi}_{\epsilon}(t)\right|^{2}}{b_{n}\left(\inf _{|t| \leq 2 b_{n}^{-1}}\left|f_{\epsilon}^{\mathrm{ft}}(t)\right|\right)^{2}}\right)$, and (A.16) follows by (B.5), (B.6), Assumptions O and O'. 
Let $a_{j}=\left(Y_{j}, X_{j}, Z_{j}, X_{j}^{r}, Z_{j}^{r}\right)$ and

$$
\hat{p}_{n}\left(a_{j}, a_{k}, a_{l}\right)=\left\{\begin{array}{c}
\hat{q}_{n}\left(a_{j}, a_{k}, a_{l}\right)+\hat{q}_{n}\left(a_{j}, a_{l}, a_{k}\right)+\hat{q}_{n}\left(a_{k}, a_{j}, a_{l}\right) \\
+\hat{q}_{n}\left(a_{l}, a_{j}, a_{k}\right)+\hat{q}_{n}\left(a_{k}, a_{l}, a_{j}\right)+\hat{q}_{n}\left(a_{l}, a_{k}, a_{j}\right)
\end{array}\right\}
$$

where

$\hat{q}_{n}\left(a_{j}, a_{k}, a_{l}\right)=\frac{1}{6 b_{n}^{3}} \iint\left\{+\frac{1}{2 \pi} \int\left\{\begin{array}{c}\mathbb{K}_{\epsilon}\left(\frac{x-X_{j}}{b_{n}}\right) Y_{k} \mathbb{K}_{\epsilon}\left(\frac{x-X_{k}}{b_{n}}\right) \mathbb{K}_{\nu}\left(\frac{z-Z_{k}}{b_{n}}\right) \\ -Y_{j} \mathbb{K}_{\epsilon}\left(\frac{x-X_{j}}{b_{n}}\right) \mathbb{K}_{\epsilon}\left(\frac{x-X_{k}}{b_{n}}\right) \mathbb{K}_{\nu}\left(\frac{z-Z_{k}}{b_{n}}\right) \\ +Y_{j} e^{-\mathrm{i} t\left(\frac{x-X_{j}}{b_{n}}\right)} Y_{k} \mathbb{K}_{\epsilon}\left(\frac{x-X_{k}}{b_{n}}\right) \mathbb{K}_{\nu}\left(\frac{z-Z_{k}}{b_{n}}\right) \\ \left.+\frac{x-X_{j}}{b_{n}}\right) Y_{k} e^{-\mathrm{i} t\left(\frac{x-X_{k}}{b_{n}}\right)} \mathbb{K}_{\nu}\left(\frac{z-Z_{k}}{b_{n}}\right) \\ -Y_{\nu}\left(\frac{z-Z_{k}}{b_{n}}\right) \\ +\frac{1}{2 \pi} \int\left\{\begin{array}{c}\mathbb{K}_{\epsilon}\left(\frac{x-X_{j}}{b_{n}}\right) e^{-\mathrm{i} t\left(\frac{x-X_{k}}{b_{n}}\right)} \mathbb{K}_{\nu}\left(\frac{z-Z_{k}}{b_{n}}\right) \\ \mathbb{K}_{\epsilon}\left(\frac{x-X_{j}}{b_{n}}\right) Y_{k} \mathbb{K}_{\epsilon}\left(\frac{x-X_{k}}{b_{n}}\right) e^{-\mathrm{i} t\left(\frac{z-Z_{k}}{b_{n}}\right)} \\ -Y_{j} \mathbb{K}_{\epsilon}\left(\frac{x-X_{j}}{b_{n}}\right) \mathbb{K}_{\epsilon}\left(\frac{x-X_{k}}{b_{n}}\right) e^{-\mathrm{it}\left(\frac{z-Z_{k}}{b_{n}}\right)}\end{array}\right\} \frac{\Pi_{\nu, l}\left(t / b_{n}\right) K^{\mathrm{ft}(t)}}{\left.f_{\epsilon}^{\mathrm{ft}\left(t / b_{n}\right)}\right) K^{\mathrm{ft}}(t)} d t\end{array}\right\} e^{\mathrm{i}(x, z) \xi} d x d z\right.$

Further decompose

$$
\hat{S}_{n}(\xi)=n^{-2}(n-1)(n-2) \hat{U}_{n}(\xi)+S_{1, n}(\xi)+\cdots+S_{4, n}(\xi),
$$

where

$$
\begin{aligned}
\hat{U}_{n}(\xi) & =\left(\begin{array}{l}
n \\
3
\end{array}\right) \sum_{j=1}^{-1} \sum_{k=j+1}^{n-2} \sum_{l=k+1}^{n} p_{n}\left(a_{j}, a_{k}, a_{l}\right), \\
S_{1, n}(\xi) & =\frac{6}{n^{3}} \sum_{j=1}^{n-1} \sum_{k=j+1}^{n}\left[p_{n}\left(a_{j}, a_{k}, a_{j}\right)+p_{n}\left(a_{k}, a_{j}, a_{k}\right)\right], S_{2, n}(\xi)=\frac{6}{n^{3}} \sum_{j=1}^{n-1} \sum_{k=j+1}^{n}\left[p_{n}\left(a_{k}, a_{j}, a_{j}\right)+p_{n}\left(a_{j}, a_{k}, a_{k}\right)\right], \\
S_{3, n}(\xi) & =\frac{6}{n^{3}} \sum_{j=1}^{n-1} \sum_{k=j+1}^{n}\left[p_{n}\left(a_{j}, a_{j}, a_{k}\right)+p_{n}\left(a_{k}, a_{k}, a_{j}\right)\right], S_{4, n}(\xi)=\frac{6}{n^{3}} \sum_{j=1}^{n} p_{n}\left(a_{j}, a_{j}, a_{j}\right) .
\end{aligned}
$$

We claim that $S_{1, n}(\xi), \ldots, S_{4, n}(\xi)$ are asymptotically negligible, i.e.

$$
\sup _{\xi \in \Xi}\left|S_{1, n}(\xi)\right|, \ldots, \sup _{\xi \in \Xi}\left|S_{4, n}(\xi)\right|=o_{p}\left(n^{-1 / 2}\right) .
$$


As $S_{3, n}(\xi)=S_{4, n}(\xi)=0$, we focus on $S_{1, n}(\xi)$ and $S_{2, n}(\xi)$. Note that

$$
\begin{aligned}
& \hat{q}_{n}\left(a_{j}, a_{k}, a_{l}\right)=\frac{Y_{k}-Y_{j}}{12 \pi b_{n}} \iiint\left\{\begin{array}{c}
\left\{\frac{1}{2 \pi} \int e^{-\mathrm{i}\left(t_{1}+t_{2}-b_{n} \xi_{1}\right) \tilde{x}} d \tilde{x}\right\}\left\{\frac{1}{2 \pi} \int e^{-\mathrm{i}\left(t_{3}-b_{n} \xi_{2}\right) \tilde{z}} d \tilde{z}\right\} \\
\times \frac{K^{\mathrm{ft}}\left(t_{1}\right) K^{\mathrm{ft}}\left(t_{2}\right) K^{\mathrm{ft}}\left(t_{3}\right) e^{\mathrm{i}\left(\frac{t_{1} X_{j}+t_{2} X_{k}+t_{3} z_{k}}{b_{n}}\right)}}{f_{\epsilon}^{\mathrm{ft}}\left(t_{1} / b_{n}\right) f_{\epsilon}^{\mathrm{ft}}\left(t_{2} / b_{n}\right) f_{\nu}^{\mathrm{ft}}\left(t_{3} / b_{n}\right)} \\
\times\left\{1+\Pi_{\epsilon, l}\left(t_{1} / b_{n}\right)+\Pi_{\epsilon, l}\left(t_{2} / b_{n}\right)+\Pi_{\nu, l}\left(t_{3} / b_{n}\right)\right\}
\end{array}\right\} d t_{1} d t_{2} d t_{3} \\
& =\frac{Y_{k}-Y_{j}}{12 \pi b_{n}}\left\{\begin{array}{c}
\int \frac{K^{\mathrm{ft}}(t) K^{\mathrm{ft}}\left(-t+b_{n} \xi_{1}\right) e^{\mathrm{it}\left(\frac{X_{j}-X_{k}}{b_{n}}\right)}}{f_{\epsilon}^{\mathrm{ft}}\left(t / b_{n}\right) f_{\epsilon}^{\mathrm{ft}}\left(-t / b_{n}+\xi_{1}\right)} d t \frac{K^{\mathrm{ft}}\left(b_{n} \xi_{2}\right)}{f_{\nu}^{\mathrm{ft}}\left(\xi_{2}\right)} e^{\mathrm{i}\left(X_{k}, Z_{k}\right) \xi} \\
\times\left\{1+\Pi_{\epsilon, l}\left(t / b_{n}\right)+\Pi_{\epsilon, l}\left(-t / b_{n}+\xi_{1}\right)+\Pi_{\nu, l}\left(\xi_{2}\right)\right\}
\end{array}\right\},
\end{aligned}
$$

where the first equality follows from the change of variables $\tilde{x}=\frac{x}{b_{n}}$ and $\tilde{z}=\frac{z}{b_{n}}$ and the second equality follows by the property of the Dirac delta function, which implies

$$
S_{1, n}(\xi), S_{2, n}(\xi)=O_{p}\left(\frac{\sup _{|t| \leq 2 b_{n}^{-1}}\left|\Pi_{\epsilon, l}(t)\right|}{n b_{n}\left(\inf _{|t| \leq 2 b_{n}^{-1}}\left|f_{\epsilon}^{\mathrm{ft}}(t)\right|\right)^{2}}\right)
$$

and (A.17) follows by (A.18), Assumptions O and O', and Lemma 10.

Thus, our focus turns to $\hat{U}_{n}(\xi)$. Let $\hat{U}_{n}^{*}(\xi)=E\left[\hat{r}_{n}\left(a_{1} ; \xi\right)\right]+\frac{3}{n} \sum_{j=1}^{n}\left\{\hat{r}_{n}\left(a_{j} ; \xi\right)-E\left[\hat{r}_{n}\left(a_{j} ; \xi\right)\right]\right\}$ for $\hat{r}_{n}\left(a_{j}\right)=E\left[\hat{p}_{n}\left(a_{j}, a_{k}, a_{l}\right) \mid a_{j}\right]$. By the Hájek projection (see, for example, Ahn and Powell, 1993, Lemma A.3), if

$$
E\left[\sup _{\xi \in \Xi} \hat{p}_{n}\left(a_{j}, a_{k}, a_{l}\right)^{2}\right]=o(n)
$$

then

$$
\sup _{\xi \in \Xi}\left|\hat{U}_{n}(\xi)-\hat{U}_{n}^{*}(\xi)\right|=o_{p}\left(n^{-1 / 2}\right)
$$

To show (A.19), note that

$$
\begin{aligned}
& E\left[\sup _{\xi \in \Xi} \hat{p}_{n}\left(a_{j}, a_{k}, a_{l}\right)^{2}\right] \leq 6 E\left[\sup _{\xi \in \Xi} \hat{q}_{n}\left(a_{j}, a_{k}, a_{l}\right)^{2}\right]
\end{aligned}
$$

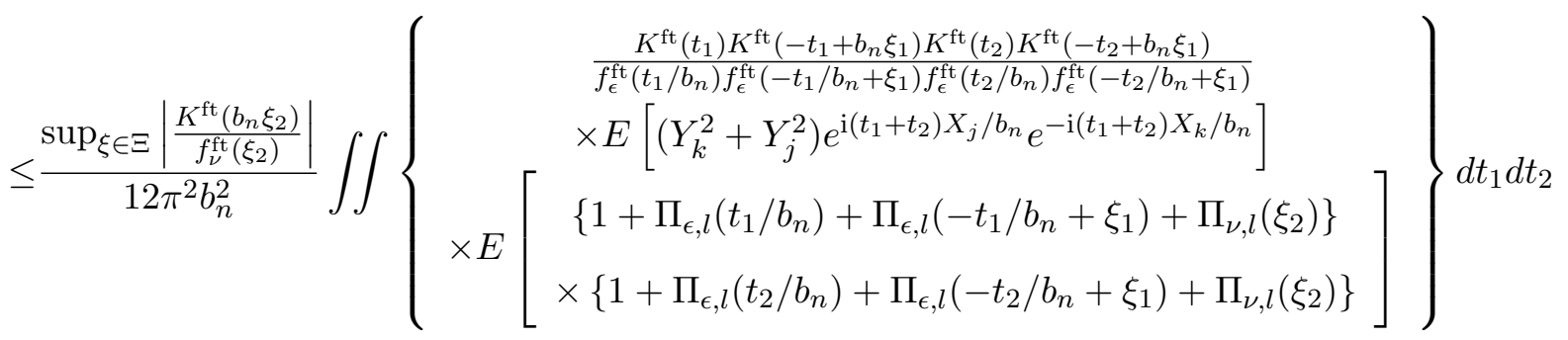

$$
\begin{aligned}
& =O\left(\frac{\left(\sup _{|t| \leq 2 b_{n}^{-1}}\left|\Pi_{\epsilon, l}(t)\right|\right)^{2}}{b_{n}^{2}\left(\inf _{|t| \leq 2 b_{n}^{-1}}\left|f_{\epsilon}^{\mathrm{ft}}(t)\right|\right)^{4}}\right),
\end{aligned}
$$


where the first step follows from Jensen's inequality and the last step follows by the boundedness of $E\left[Y^{2} \mid X^{*}\right]$ guaranteed by Assumption D (i). Then, (A.19) follows by (A.21), Assumptions O and O', and Lemma 10.

Decompose $\hat{r}_{n}\left(a_{j} ; \xi\right)=\hat{r}_{n}^{*}\left(a_{j} ; \xi\right)+\hat{r}_{n}^{\epsilon}\left(a_{j} ; \xi\right)+\hat{r}_{n}^{\nu}\left(a_{j} ; \xi\right)$, where

$$
\begin{aligned}
& \hat{r}_{n}^{*}\left(a_{j} ; \xi\right)=\iint \frac{e^{\mathrm{i}(x, z) \xi}}{3 b_{n}^{3}}\left\{\begin{array}{c}
\mathbb{K}_{\epsilon}\left(\frac{x-X_{j}}{b_{n}}\right) E\left[Y K\left(\frac{x-X^{*}}{b_{n}}\right) K\left(\frac{z-Z^{*}}{b_{n}}\right)\right] \\
-Y_{j} \mathbb{K}_{\epsilon}\left(\frac{x-X_{j}}{b_{n}}\right) E\left[K\left(\frac{x-X^{*}}{b_{n}}\right) K\left(\frac{z-Z^{*}}{b_{n}}\right)\right] \\
+E\left[K\left(\frac{x-X^{*}}{b_{n}}\right)\right] Y_{j} \mathbb{K}_{\epsilon}\left(\frac{x-X_{j}}{b_{n}}\right) \mathbb{K}_{\nu}\left(\frac{z-Z_{j}}{b_{n}}\right) \\
-E\left[Y K\left(\frac{x-X^{*}}{b_{n}}\right)\right] \mathbb{K}_{\epsilon}\left(\frac{x-X_{j}}{b_{n}}\right) \mathbb{K}_{\nu}\left(\frac{z-Z_{j}}{b_{n}}\right) \\
+E\left[K\left(\frac{x-X^{*}}{b_{n}}\right)\right] E\left[Y K\left(\frac{x-X^{*}}{b_{n}}\right) K\left(\frac{z-Z^{*}}{b_{n}}\right)\right] \\
-E\left[Y K\left(\frac{x-X^{*}}{b_{n}}\right)\right] E\left[K\left(\frac{x-X^{*}}{b_{n}}\right) K\left(\frac{z-Z^{*}}{b_{n}}\right)\right]
\end{array}\right\} d x d z, \\
& \hat{r}_{n}^{\epsilon}\left(a_{j} ; \xi\right)=\iiint \frac{\Pi_{\epsilon, j}\left(t / b_{n}\right) K^{\mathrm{ft}}(t) e^{\mathrm{i}(x, z) \xi}}{6 \pi b_{n}^{3}}\left\{\begin{array}{c}
E\left[e^{\left.-\mathrm{i} t\left(\frac{x-X^{*}}{b_{n}}\right)\right]} E\left[Y K\left(\frac{x-X^{*}}{b_{n}}\right) K\left(\frac{z-Z^{*}}{b_{n}}\right)\right]\right. \\
-E\left[Y e^{-\mathrm{i} t\left(\frac{x-X^{*}}{b_{n}}\right)}\right] E\left[K\left(\frac{x-X^{*}}{b_{n}}\right) K\left(\frac{z-Z^{*}}{b_{n}}\right)\right] \\
+E\left[K\left(\frac{x-X^{*}}{b_{n}}\right)\right] E\left[Y e^{-\mathrm{i} t\left(\frac{x-X^{*}}{b_{n}}\right)} K\left(\frac{z-Z^{*}}{b_{n}}\right)\right] \\
-E\left[Y K\left(\frac{x-X^{*}}{b_{n}}\right)\right] E\left[e^{-\mathrm{i} t\left(\frac{x-X^{*}}{b_{n}}\right)} K\left(\frac{z-Z^{*}}{b_{n}}\right)\right]
\end{array}\right\} d t d x d z, \\
& \hat{r}_{n}^{\nu}\left(a_{j} ; \xi\right)=\iiint \frac{\Pi_{\nu, j}\left(t / b_{n}\right) K^{\mathrm{ft}}(t) e^{\mathrm{i}(x, z) \xi}}{6 \pi b_{n}^{3}}\left\{\begin{array}{c}
E\left[K\left(\frac{x-X^{*}}{b_{n}}\right)\right] E\left[Y K\left(\frac{x-X^{*}}{b_{n}}\right) e^{-\mathrm{i} t\left(\frac{z-Z^{*}}{b_{n}}\right)}\right] \\
-E\left[Y K\left(\frac{x-X^{*}}{b_{n}}\right)\right] E\left[K\left(\frac{x-X^{*}}{b_{n}}\right) e^{-\mathrm{i} t\left(\frac{z-Z^{*}}{b_{n}}\right)}\right]
\end{array}\right\} d t d x d z,
\end{aligned}
$$

which are obtained using Lemma 2.

For $\hat{U}_{n}^{*}(\xi)$, we first claim that it has the same bias term as $U_{n}^{*}(\xi)$, i.e.

$$
E\left[\hat{r}_{n}\left(a_{j} ; \xi\right)\right]=E\left[r_{n}\left(d_{j} ; \xi\right)\right]
$$

which follows from $E\left[\hat{r}_{n}^{\epsilon}\left(a_{j} ; \xi\right)\right]=E\left[\hat{r}_{n}^{\nu}\left(a_{j} ; \xi\right)\right]=0$ and $E\left[\hat{r}_{n}^{*}\left(a_{j} ; \xi\right)\right]=E\left[r_{n}\left(d_{j} ; \xi\right)\right]$. Here, $E\left[\hat{r}_{n}^{\epsilon}\left(a_{j} ; \xi\right)\right]=$ $E\left[\hat{r}_{n}^{\nu}\left(a_{j} ; \xi\right)\right]=0$ is an immediate result of $E\left[\Pi_{\epsilon, j}\left(t / b_{n}\right)\right]=E\left[\Pi_{\nu, j}\left(t / b_{n}\right)\right]=0$, and $E\left[\hat{r}_{n}^{*}\left(a_{j} ; \xi\right)\right]=$ $E\left[r_{n}\left(d_{j} ; \xi\right)\right]$ is shown by using Lemma 2 .

For the main term in $\hat{U}_{n}^{*}(\xi)$, we first note that

$$
3\left\{\hat{r}_{n}^{*}\left(a_{j} ; \xi\right)-E\left[\hat{r}_{n}^{*}\left(a_{j} ; \xi\right)\right]\right\}=2\left\{r_{n}\left(d_{j} ; \xi\right)-E\left[r_{n}\left(d_{j} ; \xi\right)\right]\right\}
$$


Also note that

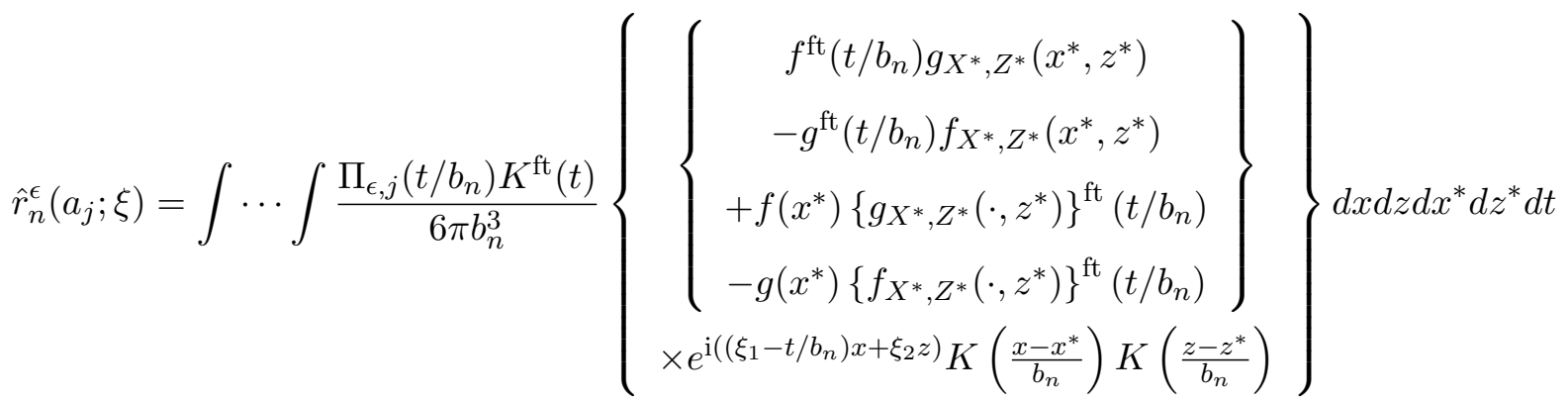

$$
\begin{aligned}
& =\int \frac{\Pi_{\epsilon, j}\left(t / b_{n}\right) K^{\mathrm{ft}}(t)}{6 \pi b_{n}}\left\{\left\{\begin{array}{c}
f^{\mathrm{ft}}\left(t / b_{n}\right) \iint e^{\mathrm{i}\left\{\left(\xi_{1}-t / b_{n}\right) x^{*}+\xi_{2} z^{*}\right\}} g_{X^{*}, Z^{*}}\left(x^{*}, z^{*}\right) d x^{*} d z^{*} \\
-g^{\mathrm{ft}}\left(t / b_{n}\right) \iint e^{\mathrm{i}\left\{\left(\xi_{1}-t / b_{n}\right) x^{*}+\xi_{2} z^{*}\right\}} f_{X^{*}, Z^{*}}\left(x^{*}, z^{*}\right) d x^{*} d z^{*} \\
+\iint e^{\mathrm{i}\left(\left(\xi_{1}-t / b_{n}\right) x^{*}+\xi_{2} z^{*}\right)} f\left(x^{*}\right)\left\{g_{X^{*}, Z^{*}}\left(\cdot, z^{*}\right)\right\}^{\mathrm{ft}}\left(t / b_{n}\right) d x^{*} d z^{*} \\
-\iint e^{\mathrm{i}\left(\left(\xi_{1}-t / b_{n}\right) x^{*}+\xi_{2} z^{*}\right)} g\left(x^{*}\right)\left\{f_{X^{*}, Z^{*}}\left(\cdot, z^{*}\right)\right\}^{\mathrm{ft}}\left(t / b_{n}\right) d x^{*} d z^{*}
\end{array}\right\} d t\right. \\
& =\int \frac{\Pi_{\epsilon, j}(t) K^{\mathrm{ft}}\left(t b_{n}\right) K^{\mathrm{ft}}\left(\left(\xi_{1}-t\right) b_{n}\right) K^{\mathrm{ft}}\left(\xi_{2} b_{n}\right)}{6 \pi}\left\{\begin{array}{c}
f^{\mathrm{ft}}(t) g_{X^{*}, Z^{*}}^{\mathrm{ft}}\left(\xi_{1}-t, \xi_{2}\right)-g^{\mathrm{ft}}(t) f_{X^{*}, Z^{*}}^{\mathrm{ft}}\left(\xi_{1}-t, \xi_{2}\right) \\
+f^{\mathrm{ft}}\left(\xi_{1}-t\right) g_{X^{*}, Z^{*}}^{\mathrm{ft}}\left(t, \xi_{2}\right)-g^{\mathrm{ft}}\left(\xi_{1}-t\right) f_{X^{*}, Z^{*}}^{\mathrm{ft}}\left(t, \xi_{2}\right)
\end{array}\right\} d t,
\end{aligned}
$$

where the first equality follows by Lemma 11 and the second equality follows from the change of variables $\tilde{x}=\left(x-x^{*}\right) / b_{n}$ and $\tilde{z}=\left(z-z^{*}\right) / b_{n}$, which, by using the dominant convergence theorem, implies

$$
\sup _{\xi \in \Xi}\left|\hat{r}_{n}^{\epsilon}\left(a_{j} ; \xi\right)-\int \frac{\Pi_{\epsilon, j}(t)}{6 \pi}\left\{\begin{array}{c}
f^{\mathrm{ft}}(t) g_{X^{*}, Z^{*}}^{\mathrm{ft}}\left(\xi_{1}-t, \xi_{2}\right)-g^{\mathrm{ft}}(t) f_{X^{*}, Z^{*}}^{\mathrm{ft}}\left(\xi_{1}-t, \xi_{2}\right) \\
+f^{\mathrm{ft}}\left(\xi_{1}-t\right) g_{X^{*}, Z^{*}}^{\mathrm{ft}}\left(t, \xi_{2}\right)-g^{\mathrm{ft}}\left(\xi_{1}-t\right) f_{X^{*}, Z^{*}}^{\mathrm{ft}}\left(t, \xi_{2}\right)
\end{array}\right\} d t\right|=o_{p}(1) .
$$

By similar arguments, we have

$$
\sup _{\xi \in \Xi}\left|\hat{r}_{n}^{\nu}\left(a_{j} ; \xi\right)-\frac{\Pi_{\nu, j}\left(\xi_{2}\right)}{6 \pi} \int\left\{\begin{array}{c}
f^{\mathrm{ft}}(t) g_{X^{*}, Z^{*}}^{\mathrm{ft}}\left(\xi_{1}-t, \xi_{2}\right) \\
-g^{\mathrm{ft}}(t) f_{X^{*}, Z^{*}}^{\mathrm{ft}}\left(\xi_{1}-t, \xi_{2}\right)
\end{array}\right\} d t\right|=o_{p}(1) .
$$

Then, by Theorem 3.9 of Chen and White (1998) and Assumption O', we have

$$
\frac{3}{\sqrt{n}} \sum_{j=1}^{n}\left\{\hat{r}_{n}\left(a_{j} ; \xi\right)-E\left[\hat{r}_{n}\left(a_{j} ; \xi\right)\right]\right\} \Rightarrow \hat{G}^{\mathrm{os}}(\xi)
$$


Combining (A.16), (A.17), (A.20), (A.22), and (A.23), if $\mathrm{H}_{0}$ holds, (A.4) implies

$$
\sqrt{n} \hat{T}_{n}(\xi) \Rightarrow \hat{G}^{\mathrm{os}}(\xi),
$$

and if $\mathrm{H}_{1 n}$ holds, (A.13) implies

$$
\sqrt{n} \hat{T}_{n}(\xi) \Rightarrow \hat{G}^{\mathrm{os}}(\xi)+\mu_{\Delta}(\xi) .
$$

\section{A.6 Proof of Theorem 6}

This proof follows along the same lines as Theorem 5, except that (A.16) follows by (??), (??), (B.5), (B.6), Assumptions S and S'. Also (A.17) follows by (A.18), Assumptions S and S', and Lemma 10. Finally, (A.19) follows by (A.21), Assumptions S and S', and Lemma 10.

Then, by Theorem 3.9 of Chen and White (1998) and Assumption S', we have

$$
\frac{3}{\sqrt{n}} \sum_{j=1}^{n}\left\{\hat{r}_{n}\left(a_{j} ; \xi\right)-E\left[\hat{r}_{n}\left(a_{j} ; \xi\right)\right]\right\} \Rightarrow \hat{G}^{\mathrm{ss}}(\xi) .
$$

Combining (A.16), (A.17), (A.20), (A.22), and (A.24), if $\mathrm{H}_{0}$ holds, (A.4) implies

$$
\sqrt{n} \hat{T}_{n}(\xi) \Rightarrow \hat{G}^{\mathrm{ss}}(\xi),
$$

and if $\mathrm{H}_{1 n}$ holds, (A.13) implies

$$
\sqrt{n} \hat{T}_{n}(\xi) \Rightarrow \hat{G}^{\mathrm{ss}}(\xi)+\mu_{\Delta}(\xi) .
$$




\section{B Lemmas}

Lemma 1. For a random error $\eta$ with $f_{\eta}^{\mathrm{ft}}(t) \neq 0$ for all $t \in \mathbb{R}$, we have

$$
\begin{aligned}
\int \mathbb{K}_{\eta}(u) e^{\mathrm{i} b_{n} u \xi} d u & =\frac{K^{\mathrm{ft}}\left(b_{n} \xi\right)}{f_{\eta}^{\mathrm{ft}}(\xi)} \\
\int \mathbb{K}_{\eta}(u+c) \mathbb{K}_{\eta}(u) e^{\mathrm{i} b_{n} u \xi} d u & =\frac{1}{2 \pi} \int \frac{e^{-\mathrm{i} t c} K^{\mathrm{ft}}(t) K^{\mathrm{ft}}\left(-t+b_{n} \xi\right)}{f_{\eta}^{\mathrm{ft}}\left(t / b_{n}\right) f_{\eta}^{\mathrm{ft}}\left(-t / b_{n}+\xi\right)} d t
\end{aligned}
$$

Proof. The conclusion follows by using $\int \delta(u-b) m(u) d u=m(b)$ with the Dirac delta function $\delta(u) \equiv$ $\frac{1}{2 \pi} \int e^{-\mathrm{i} u t} d t$ for

$$
\begin{aligned}
\int \mathbb{K}_{\eta}(u) e^{\mathrm{i} b_{n} u \xi} d u & =\int\left(\frac{1}{2 \pi} \int e^{-\mathrm{i} t u} \frac{K^{\mathrm{ft}}(t)}{f_{\eta}^{\mathrm{ft}}\left(t / b_{n}\right)} d t\right) e^{\mathrm{i} b_{n} u \xi} d u \\
& =\int\left(\frac{1}{2 \pi} \int e^{-\mathrm{i}\left(t-b_{n} \xi\right) u} d u\right) \frac{K^{\mathrm{ft}}(t)}{f_{\eta}^{\mathrm{ft}}\left(t / b_{n}\right)} d t \\
\int \mathbb{K}_{\eta}(u+c) \mathbb{K}_{\eta}(u) e^{\mathrm{i} b_{n} u \xi} d u & =\int\left(\frac{1}{2 \pi} \int e^{-\mathrm{i} t_{1}(u+c)} \frac{K^{\mathrm{ft}}\left(t_{1}\right)}{f_{\eta}^{\mathrm{ft}}\left(t_{1} / b_{n}\right)} d t_{1}\right)\left(\frac{1}{2 \pi} \int e^{-\mathrm{i} t_{2} u} \frac{K^{\mathrm{ft}}\left(t_{2}\right)}{f_{\eta}^{\mathrm{ft}}\left(t_{2} / b_{n}\right)} d t_{2}\right) e^{\mathrm{i} b_{n} u \xi} d u \\
& =\frac{1}{2 \pi} \iint\left(\frac{1}{2 \pi} \int e^{-\mathrm{i}\left(t_{1}+t_{2}-b_{n} \xi\right) u} d u\right) \frac{e^{-\mathrm{i} t_{1} c} K^{\mathrm{ft}}\left(t_{1}\right) K^{\mathrm{ft}}\left(t_{2}\right)}{f_{\eta}^{\mathrm{ft}}\left(t_{1} / b_{n}\right) f_{\eta}^{\mathrm{ft}}\left(t_{2} / b_{n}\right)} d t_{1} d t_{2} .
\end{aligned}
$$

Lemma 2. Under Assumption D, for $\left\{k_{1}, k_{2}\right\} \in\{0,1\}$, we have

$$
E\left[Y^{k_{1}} \mathbb{K}_{\epsilon}\left(\frac{x-X}{b_{n}}\right) \mathbb{K}_{\nu}^{k_{2}}\left(\frac{z-Z}{b_{n}}\right)\right]=E\left[Y^{k_{1}} K\left(\frac{x-X^{*}}{b_{n}}\right) K^{k_{2}}\left(\frac{z-Z^{*}}{b_{n}}\right)\right] .
$$

Proof. As the proofs are similar, we focus on the case $k_{1}=k_{2}=1$. The result follows by

$$
\begin{aligned}
& E\left[Y \mathbb{K}_{\epsilon}\left(\frac{x-X}{b_{n}}\right) \mathbb{K}_{\nu}\left(\frac{z-Z}{b_{n}}\right)\right] \\
= & \frac{1}{4 \pi^{2}} \iint e^{-\mathrm{i}\left(t_{1} x+t_{2} z\right) / b_{n}}\left\{E[Y \mid X, Z] f_{X, Z}\right\}^{\mathrm{ft}}\left(t_{1} / b_{n}, t_{2} / b_{n}\right) \frac{K^{\mathrm{ft}}\left(t_{1}\right) K^{\mathrm{ft}}\left(t_{2}\right)}{f_{\epsilon}^{\mathrm{ft}}\left(t_{1} / b_{n}\right) f_{\nu}^{\mathrm{ft}}\left(t_{2} / b_{n}\right)} d t_{1} d t_{2} \\
= & \frac{1}{4 \pi^{2}} \iint e^{-\mathrm{i}\left(t_{1} x+t_{2} z\right) / b_{n}} g_{X^{*}, Z^{*}}^{\mathrm{ft}}\left(t_{1} / b_{n}, t_{2} / b_{n}\right) K^{\mathrm{ft}}\left(t_{1}\right) K^{\mathrm{ft}}\left(t_{2}\right) d t_{1} d t_{2} \\
= & E\left[Y K\left(\frac{x-X^{*}}{b_{n}}\right) K\left(\frac{z-Z^{*}}{b_{n}}\right)\right]
\end{aligned}
$$

where the second step uses Assumption D (ii) and the last step follows by the convolution theorem. 
Lemma 3. Under Assumption $D$ and $O / S$, we have

$$
\begin{aligned}
& \iint\left\{b_{n}^{-3} E\left[Y K\left(\frac{x-X^{*}}{b_{n}}\right)\right] E\left[K\left(\frac{x-X^{*}}{b_{n}}\right) K\left(\frac{z-Z^{*}}{b_{n}}\right)\right]-g(x) f_{X^{*}, Z^{*}}(x, z)\right\} e^{\mathrm{i}(x, z) \xi} d x d z=\tau_{1, n}, \\
& \iint\left\{b_{n}^{-3} E\left[K\left(\frac{x-X^{*}}{b_{n}}\right)\right] E\left[Y K\left(\frac{x-X^{*}}{b_{n}}\right) K\left(\frac{z-Z^{*}}{b_{n}}\right)\right]-f(x) g_{X^{*}, Z^{*}}(x, z)\right\} e^{\mathrm{i}(x, z) \xi} d x d z=\tau_{2, n},
\end{aligned}
$$

where $\tau_{k, n}=O\left(b_{n}^{p}\right)$ under Assumption $O$ and $\tau_{k, n}=0$ under Assumption $S$ for $k=1,2$.

Proof. As the proofs are similar, we focus on the statement concerning $\tau_{2, n}$. Define

$$
J_{1, n}(x)=b_{n}^{-1} E\left[K\left(\frac{x-X^{*}}{b_{n}}\right)\right], \quad J_{2, n}(x, z)=b_{n}^{-2} E\left[Y K\left(\frac{x-X^{*}}{b_{n}}\right) K\left(\frac{z-Z^{*}}{b_{n}}\right)\right] .
$$

Under Assumption S, $f$ and $g_{X^{*}, Z^{*}}$ are both infinitely differentiable and $K$ is an infinite-order kernel, which implies $J_{1, n}(x)=f(x)$ and $J_{2, n}(x, z)=g_{X^{*}, Z^{*}}(x, z)$.

Under Assumption $\mathrm{O}, f$ and $g_{X^{*}, Z^{*}}$ are both $p$-times continuously differentiable and $K$ is a $p^{\text {th }}$ order kernel, which implies $J_{1, n}(x)=f(x)+b_{n}^{p} \Delta_{1, n}(x)$ and $J_{2, n}(x, z)=g_{X^{*}, Z^{*}}(x, z)+b_{n}^{p} \Delta_{2, n}(x, z)$, where $\Delta_{1, n}(x)=\frac{1}{p !} \int f^{(p)}(\bar{x}) K\left(x^{*}\right) x^{* p} d x^{*}$ for some $\bar{x}$ lying between $x$ and $x-b_{n} \tilde{x}^{*}$ and $\Delta_{2, n}(x, z)=$ $\sum_{l=1}^{p} \frac{1}{l !(p-l) !} \iint g_{X^{*}, Z^{*}}^{(l, p-l)}(\overline{\bar{x}}, \overline{\bar{z}}) K\left(x^{*}\right) K\left(z^{*}\right) x^{* l} z^{* p-l} d x^{*} d z^{*}$ for some $(\overline{\bar{x}}, \overline{\bar{z}})$ lying between $(x, z)$ and $(x-$ $\left.b_{n} \tilde{x}^{*}, z-b_{n} \tilde{z}^{*}\right)$. The Lipschitz condition on $f^{(p)}$ and $g_{X^{*}, Z^{*}}^{(l, p-l)}$ as assumed in Assumption O (i) implies

$$
\begin{aligned}
\left|\Delta_{1, n}(x)\right| & \leq \frac{1}{p !}\left\{\mu_{K}^{p}\left|f^{(p)}(x)\right|+b_{n} \mu_{K}^{p+1}\left|m_{1, p}(x)\right|\right\} \\
\left|\Delta_{2, n}(x, z)\right| & \leq \sum_{l=1}^{p} \frac{1}{l !(p-l) !}\left\{\mu_{K}^{l} \mu_{K}^{p-l}\left|g_{X^{*}, Z^{*}}^{(l, p-l)}(x, z)\right|+b_{n}\left(\mu_{K}^{l+1} \mu_{K}^{p-l}+\mu_{K}^{l} \mu_{K}^{p+1-l}\right)\left|m_{4, p}(x, z)\right|\right\},
\end{aligned}
$$

where $\mu_{K}^{d}=\int K(u)|u|^{d} d u$. Then, the conclusion follows by

$$
\begin{gathered}
\iint\left\{J_{1, n}(x) J_{2, n}(x, z)-f(x) g_{X^{*}, Z^{*}}(x, z)\right\} e^{\mathrm{i}(x, z) \xi} d x d z \\
=\iint\left\{\begin{array}{c}
\left\{J_{1, n}-f\right\}(x) g_{X^{*}, Z^{*}}(x, z) \\
+f(x)\left\{J_{2, n}-g_{X^{*} \cdot Z^{*}}\right\}(x, z) \\
+\left\{J_{1, n}-f\right\}(x)\left\{J_{2, n}-g_{X^{*} . Z^{*}}\right\}(x, z)
\end{array}\right\} e^{\mathrm{i}(x, z) \xi} d x d z,
\end{gathered}
$$

and the boundedness and the integrability of $f, f^{(p)}, g_{X^{*}, Z^{*}}, g_{X^{*}, Z^{*}}^{(l, p-l)}, m_{1, p}$, and $m_{4, p}$ as assumed in Assumption O (i). 


\section{Lemma 4.}

$$
\mathbb{K}_{\epsilon}(x)= \begin{cases}\sum_{h=0}^{\alpha} c_{h}^{\mathrm{os}}\left(\mathrm{i} / b_{n}\right)^{h} K^{(h)}(x) & \text { under Assumption } O \\ \sum_{h=0}^{\infty} \frac{\mu^{h}}{c^{\mathrm{ss}} h !}\left(\mathrm{i} / b_{n}\right)^{h \gamma} K^{(h \gamma)}(x) & \text { under Assumption } S\end{cases}
$$

Proof. Under Assumption O, the conclusion follows by Lemma 3.1 of Fan (1995). Under Assumption S, the conclusion follows by Lemma 5 of Dong, Otsu and Taylor (2019).

Lemma 5. Under Assumption O, we have

$$
\int \mathbb{K}_{\epsilon}(x) x^{l} e^{\mathrm{i} b_{n} x \xi_{1}} d x=\mathbb{I}\{l \leq \alpha\} c_{l}^{\mathrm{os}}\left(\xi_{1}\right) l ! b_{n}^{-l}+\mathrm{i} b_{n} \sum_{h=0}^{\alpha} \frac{c_{h}^{\mathrm{os}}(-1)^{h} \xi_{1}^{h+1}}{(h+1) !} \int K^{(h)}(x) x^{l} \bar{x}_{h}^{h+1} d x,
$$

for $l=0,1, \ldots, p$, where $\bar{x}_{h}$ lying between 0 and $x$ for $h=1, \cdots, \alpha$.

Proof. Note that

$$
\begin{aligned}
\int \mathbb{K}_{\epsilon}(x) x^{l} e^{\mathrm{i} b_{n} x \xi_{1}} d x & =\sum_{h=0}^{\alpha} c_{h}^{\mathrm{os}}\left(\mathrm{i} / b_{n}\right)^{h} \int K^{(h)}(x) x^{l} e^{\mathrm{i} b_{n} x \xi_{1}} d x \\
& =\sum_{h=0}^{\alpha} c_{h}^{\mathrm{os}}\left(\mathrm{i} / b_{n}\right)^{h}\left\{\sum_{k=0}^{h} \frac{\left(\mathrm{i} b_{n} \xi_{1}\right)^{k}}{k !} \int K^{(h)}(x) x^{l+k} d x+\frac{\left(\mathrm{i} b_{n} \xi_{1}\right)^{h+1}}{(h+1) !} \int K^{(h)}(x) x^{l} \bar{x}_{h}^{h+1} d x\right\}
\end{aligned}
$$

where the first equality follows by Lemma 4 under Assumption $\mathrm{O}$ and the second equality follows by expanding $e^{\mathrm{i} b_{n} x \xi_{1}}$ around $x=0$ to the $h$-th order and $\bar{x}_{h}$ lies between 0 and $x$ for $h=1, \ldots, \alpha$. Then, the conclusion follows by using $\int K^{(h)}(u) u^{l+k} d u=\mathbb{I}\{h=l+k\} h !(-1)^{h}$ for $h \geq l$ and $k=0,1, \ldots, \alpha$, which follows by integration-by-parts and the properties of the kernel function $K$ as assumed in Assumption O (iii).

Lemma 6. Under Assumption S, we have

$$
\int \mathbb{K}_{\epsilon}(x) x^{l} e^{\mathrm{i} b_{n} \xi_{1} x} d x=c_{l}^{\mathrm{ss}}\left(\xi_{1}\right) l ! b_{n}^{-l}
$$

Proof. Note that

$$
\begin{aligned}
\int \mathbb{K}_{\epsilon}(x) x^{l} e^{\mathrm{i} b_{n} \xi_{1} x} d x & =\sum_{h=0}^{\infty} \frac{\mu^{h}}{c^{\mathrm{ss}} h !}\left(\mathrm{i} / b_{n}\right)^{h \gamma} \int K^{(h \gamma)}(x) x^{l} e^{\mathrm{i} b_{n} \xi_{1} x} d x \\
& =\sum_{h=0}^{\infty} \frac{\mu^{h}}{c^{\mathrm{ss}} h !}\left(\mathrm{i} / b_{n}\right)^{h \gamma} \sum_{k=0}^{\infty} \frac{\left(\mathrm{i} b_{n} \xi_{1}\right)^{k}}{k !} \int K^{(h \gamma)}(x) x^{l+k} d x
\end{aligned}
$$


where the first equality follows by Lemma 4 under Assumption $\mathrm{S}$ and the second equality follows by expanding $e^{\mathrm{i} b_{n} x \xi_{1}}$ around $x=0$ to an infinite order. Then, the conclusion follows by $\int K^{(h \gamma)}(u) u^{l+k} d u=$ $\mathbb{I}\{h \gamma=l+k\}(l+k) !(-1)^{l+k}$ which follows by integration-by-parts and the properties of the kernel function $K$ as assumed in Assumption S (iii).

Lemma 7. There exist constants $A, v \geq 0$ independent of $n$ such that

$$
\sup _{Q} N\left(\mathcal{F}_{n}^{(r)},|\cdot|_{Q, 2}, \delta\right) \leq\left(\frac{A\left|F_{n}^{(r)}\right|_{Q, 2}}{\delta}\right)^{v}=O_{p}(1)
$$

for all $0<\delta \leq 1$ and where $\sup _{Q}$ is taken over all finitely discrete distributions on $\mathbb{R}^{3}$.

Proof. See Lemma 1 of Kato and Sasaki (2018). See also Corollary A.1 of Chernozhukov, Chetverikov and Kato (2014).

Lemma 8. Under Assumption D, for a random error $\eta \in\{\epsilon, \nu\}$, we have

$$
\sup _{|t| \leq \varrho_{n}}\left|\hat{f}_{\eta}^{\mathrm{ft}}(t)-f_{\eta}^{\mathrm{ft}}(t)\right|=O_{p}\left(n^{-1 / 2} \log \left(\varrho_{n}\right)^{1 / 2}\right)
$$

Proof. See Lemma 4 of Kato and Sasaki (2018).

Immediate results following Lemma 8 are

$$
\begin{gathered}
\sup _{\xi \in \Xi}\left|\hat{f}_{\nu}^{\mathrm{ft}}\left(\xi_{2}\right)-f_{\nu}^{\mathrm{ft}}\left(\xi_{2}\right)\right|=O_{p}\left(n^{-1 / 2}\right), \\
\sup _{|t| \leq b_{n}^{-1}}\left|\hat{f}_{\epsilon}^{\mathrm{ft}}(t)-f_{\epsilon}^{\mathrm{ft}}(t)\right|=O_{p}\left(n^{-1 / 2} \log \left(b_{n}^{-1}\right)^{1 / 2}\right) .
\end{gathered}
$$

Lemma 9. Under Assumption D, for a random error $\eta \in\{\epsilon, \nu\}$ and $\varrho_{n}=o\left(e^{n}\right)$, we have

$$
\begin{aligned}
& \sup _{|t| \leq \varrho_{n}}\left|\hat{\Pi}_{\eta}(t)\right|=O_{p}\left(\frac{\log \left(\varrho_{n}\right)^{1 / 2}}{n^{1 / 2}\left(\inf _{|t| \leq \varrho_{n}}\left|f_{\eta}^{\mathrm{ft}}(t)\right|\right)^{2}}\right), \\
& \sup _{|t| \leq \varrho_{n}}\left|\hat{\Pi}_{\eta}^{\mathrm{res}}(t)\right|=O_{p}\left(\frac{\log \left(\varrho_{n}\right)}{n\left(\inf _{|t| \leq \varrho_{n}}\left|f_{\eta}^{\mathrm{ft}}(t)\right|\right)^{4}}\right) .
\end{aligned}
$$

Proof. The conclusion follows by Lemma 8 and

$$
\sup _{|t| \leq \varrho_{n}}\left|\hat{\Pi}_{\eta}(t)\right| \leq \sup _{|t| \leq \varrho_{n}} \frac{\left(\left|\hat{f}_{\eta}^{\mathrm{ft}}(t)-f_{\eta}^{\mathrm{ft}}(t)\right|+2\left|f_{\eta}^{\mathrm{ft}}(t)\right|\right)\left|\hat{f}_{\eta}^{\mathrm{ft}}(t)-f_{\eta}^{\mathrm{ft}}(t)\right|}{2\left|f_{\eta}^{\mathrm{ft}}(t)\right|^{2}}=O_{p}\left(\frac{\sup _{|t| \leq \varrho_{n}}\left|\hat{f}_{\eta}^{\mathrm{ft}}(t)-f_{\eta}^{\mathrm{ft}}(t)\right|}{\left.\inf _{|t| \leq \varrho_{n}}\left|f_{\eta}^{\mathrm{ft}}(t)\right|\right)^{2}}\right)
$$




$$
\hat{\Pi}_{\eta}^{\mathrm{res}}(t) \leq \sup _{|t| \leq \varrho_{n}} \frac{\left(2\left|\hat{f}_{\eta}^{\mathrm{ft}}(t)-f_{\eta}^{\mathrm{ft}}(t)\right|+3\left|f_{\eta}^{\mathrm{ft}}(t)\right|\right)\left|\hat{f}_{\eta}^{\mathrm{ft}}(t)-f_{\eta}^{\mathrm{ft}}(t)\right|^{2}}{\left|f_{\eta}^{\mathrm{ft}}(t)\right|^{3}\left|\hat{f}_{\eta}^{\mathrm{ft}}(t)\right|}=O_{p}\left(\frac{\sup _{|t| \leq \varrho_{n}}\left|\hat{f}_{\eta}^{\mathrm{ft}}(t)-f_{\eta}^{\mathrm{ft}}(t)\right|^{2}}{\left(\inf _{|t| \leq \varrho_{n}}\left|f_{\eta}^{\mathrm{ft}}(t)\right|\right)^{4}}\right) .
$$

Immediate results following Lemma 9 are

$$
\begin{gathered}
\sup _{\xi \in \Xi}\left|\hat{\Pi}_{\nu}\left(\xi_{2}\right)\right|=O_{p}\left(n^{-1 / 2}\right), \\
\sup _{\xi \in \Xi}\left|\hat{\Pi}_{\nu}^{\mathrm{res}}\left(\xi_{2}\right)\right|=O_{p}\left(n^{-1}\right), \\
\sup _{|t| \leq 2 b_{n}^{-1}}\left|\hat{\Pi}_{\epsilon}(t)\right|= \begin{cases}O_{p}\left(n^{-1 / 2} \log \left(b_{n}^{-1}\right)^{1 / 2} b_{n}^{-2 \alpha}\right) & \text { under Assumption O } \\
O_{p}\left(n^{-1 / 2} \log \left(b_{n}^{-1}\right)^{1 / 2} e^{2^{\gamma+1}} \mu_{\epsilon} b_{n}^{-\gamma}\right) & \text { under Assumption S }\end{cases} \\
\sup _{|t| \leq 2 b_{n}^{-1}}\left|\hat{\Pi}_{\epsilon}^{\mathrm{res}}(t)\right|= \begin{cases}O_{p}\left(n^{-1} \log \left(b_{n}^{-1}\right) b_{n}^{-4 \alpha}\right) & \text { under Assumption O } \\
O_{p}\left(n^{-1} \log \left(b_{n}^{-1}\right) e^{2^{\gamma+2} \mu_{\epsilon} b_{n}^{-\gamma}}\right) & \text { under Assumption S }\end{cases}
\end{gathered}
$$

Lemma 10. Under Assumption D, we have

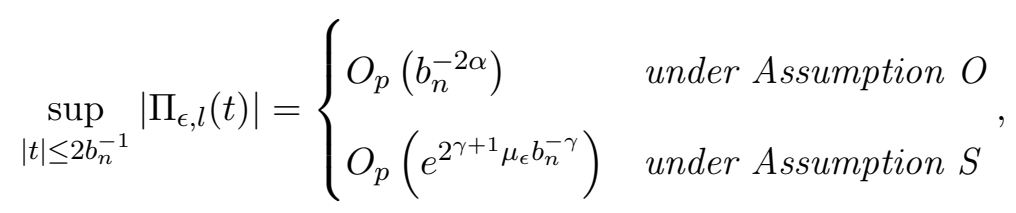

Proof. The conclusion follows by

$$
\sup _{|t| \leq 2 b_{n}^{-1}}\left|\Pi_{\epsilon, l}(t)\right| \leq \frac{\left.\sup _{|t| \leq 2 b_{n}^{-1}}\left|f_{\epsilon}^{\mathrm{ft}}(t)\right|\right)^{2}+1}{2\left(\inf _{|t| \leq 2 b_{n}^{-1}}\left|f_{\epsilon}^{\mathrm{ft}}(t)\right|\right)^{2}}=O\left(\frac{1}{\left(\inf _{|t| \leq 2 b_{n}^{-1}}\left|f_{\epsilon}^{\mathrm{ft}}(t)\right|\right)^{2}}\right)
$$

Lemma 11. Under Assumption D, we have

$$
\begin{gathered}
E\left[e^{\left.-\mathrm{i} t\left(\frac{x-X^{*}}{b_{n}}\right)\right]}=e^{-\mathrm{i} t x / b_{n}} f^{\mathrm{ft}}\left(t / b_{n}\right), E\left[Y e^{-\mathrm{i} t\left(\frac{x-X^{*}}{b_{n}}\right)}\right]=e^{-\mathrm{i} t x / b_{n}} g^{\mathrm{ft}}\left(t / b_{n}\right),\right. \\
E\left[e^{-\mathrm{i} t\left(\frac{x-X^{*}}{b_{n}}\right)} K\left(\frac{z-Z^{*}}{b_{n}}\right)\right]=e^{-\mathrm{i} t x / b_{n}} \int\left\{g_{X^{*}, Z^{*}}\left(\cdot, z^{*}\right)\right\}^{\mathrm{ft}}\left(t / b_{n}\right) K\left(\frac{z-z^{*}}{b_{n}}\right) d z^{*}, \\
E\left[Y e^{-\mathrm{i} t\left(\frac{x-X^{*}}{b_{n}}\right)} K\left(\frac{z-Z^{*}}{b_{n}}\right)\right]=e^{-\mathrm{i} t x / b_{n}} \int\left\{f_{X^{*}, Z^{*}}\left(\cdot, z^{*}\right)\right\}^{\mathrm{ft}}\left(t / b_{n}\right) K\left(\frac{z-z^{*}}{b_{n}}\right) d z^{*} .
\end{gathered}
$$


Proof. As the proofs are similar, we focus on the last statement, which follows by

$$
\begin{aligned}
E\left[e^{-\mathrm{i} t\left(\frac{x-X^{*}}{b_{n}}\right)} K\left(\frac{z-Z^{*}}{b_{n}}\right)\right] & =\iint e^{-\mathrm{i} t\left(\frac{x-x^{*}}{b_{n}}\right)} f_{X^{*}, Z^{*}}\left(x^{*}, z^{*}\right) K\left(\frac{z-z^{*}}{b_{n}}\right) d x^{*} d z^{*} \\
& =e^{-\mathrm{i} t x / b_{n}} \int\left\{\int e^{\mathrm{i} t x^{*} / b_{n}} f_{X^{*}, Z^{*}}\left(x^{*}, z^{*}\right) d x^{*}\right\} K\left(\frac{z-z^{*}}{b_{n}}\right) d z^{*} \\
& =e^{-\mathrm{i} t x / b_{n}} \int\left\{f_{X^{*}, Z^{*}}\left(\cdot, z^{*}\right)\right\}^{\mathrm{ft}}\left(t / b_{n}\right) K\left(\frac{z-z^{*}}{b_{n}}\right) d z^{*} .
\end{aligned}
$$

